\title{
Flavour and Spin of the Proton and the Meson Cloud
}

\author{
H. Holtmann ${ }^{1}$, A. Szczurek ${ }^{2}$ and J. Speth ${ }^{1}$ \\ ${ }^{1}$ Institut für Kernphysik, Forschungszentrum Jülich, \\ 53425 Jülich, Germany \\ 2 Institute of Nuclear Physics, \\ PL-31-342 Krakow, Poland
}

(September 18, 2018)

\begin{abstract}
We present a complete set of formulas for longitudinal momentum distribution functions (splitting functions) of mesons in the nucleon. It can be applied in the framework of convolution formalism to the deep-inelastic structure functions (quark distributions) of the nucleon viewed as a system composed of virtual 'mesons' and 'baryons'. Pseudoscalar and vector mesons as well as octet and decuplet baryons are included. In contrast to many approaches in the literature the present approach ensures charge and momentum conservation by the construction. We present not only spin averaged splitting functions but also helicity dependent ones, which can be used to study the spin content of the nucleon. The cut-off parameters of the underlying form factors for different vertices are determined from high-energy particle production data. We find an universal cut-off parameter for processes involving octet baryons. This information allows one to calculate the flavour and spin content of the nucleon. The value of the Gottfried Sum Rule obtained from our model $\left(S_{G}=0.224\right)$ nicely agrees with that obtained by the NMC. In addition, we calculate the $x$-dependence of the $\bar{d}-\bar{u}$ asymmetry and get an impressive
\end{abstract}


agreement with a recent fit of Martin-Stirling-Roberts. The calculated axial coupling constants for semileptonic decays of the octet baryons agree with the experimental data already with $S U(6)$ wave function for the bare nucleon. As a consequence the Bjorken Sum Rule is nicely reproduced. Although we get improvements for the Ellis-Jaffe Sum Rules for the proton and neutron in comparison to the naive quark model, the MCM is not sufficient to reproduce the experimental data.

13.30.Ce,13.60.Hb,14.20.Dh

Typeset using REVTEX 


\section{INTRODUCTION}

It is taken for granted that the nucleon consists of quarks and gluons and the underlying theory describing their interaction is QCD. However, solving the QCD equations for the many-body system at large distances is at the present time still beyond our practical abilities. Deep-inelastic scattering (DIS) of leptons is known to be a very good tool to study the structure of the proton. Here in the Bjorken limit one directly tests the quark distributions in the nucleon. Although the perturbative regime seems to be well under control and the QCD evolution equations turned out to be very successful in relating the quark distributions at different momentum scales, the nonperturbative effects are poorly known. Recent experiments on deep-inelastic scattering of leptons from nucleons [1:2] have shown the incompleteness of our understanding of the proton structure.

The violation of the Gottfried Sum Rule observed by NMC [1]

$$
S_{G}=\int_{0}^{1}\left[F_{2}^{p}(x)-F_{2}^{n}(x)\right] \frac{d x}{x}=0.24 \pm 0.016
$$

indicates that the nucleon sea is not flavour symmetric. Recent fits of quark distributions to the world data for deep-inelastic and Drell-Yan processes confirm the asymmetry [3,4]. Also the new results of the CERN dedicated NA51 experiment [5] on the dilepton production in proton-proton and proton-deuteron scattering gives evidence for the asymmetry. More detailed information can be expected from the experiments planned at Fermilab [6,7]. Perturbative effects [8] cannot explain the observed asymmetry. The dressing of the nucleon with virtual mesons provides a natural explanation for the excess of $\bar{d}$ over $\bar{u}$ quarks in the proton [9] 12] and explains the result of the NA51 experiment [13].

Another intriguing result, involving the spin structure of the proton, was found by the EMC collaboration. Their measured value of the Ellis-Jaffe Sum Rule [2] caused great excitement. After a smooth Regge extrapolation of the data they have found

$$
S_{E J}^{p}=\int_{0}^{1} g_{1}^{p}(x) d x=0.126 \pm 0.010(\text { stat }) \pm 0.015(\text { syst })
$$


This value is more then two standard deviations away from the original Ellis-Jaffe prediction $\left(S_{E J}^{p}=0.19\right)$ 14 based on the assumption of vanishing polarized strange sea. Newer experiments of the SMC [15] and SLAC [16] collaborations give a somewhat larger value for $S_{E J}^{p}$. The polarized deep inelastic scattering experiments at CERN have shown that, when supplemented with some information from semileptonic decays, only a small fraction of the proton spin is carried by valence quarks.

In view of the importance of the meson cloud for the Gottfried Sum Rule violation, one might expect that it also plays an important role for the nucleon spin. Indeed, simple estimates within the one pion exchange model seem to indicate that the meson cloud effects could contribute to the so-called spin crisis. Moreover, due to the close connection of the Ellis-Jaffe Sum Rule with semileptonic decays of octet baryons (see section VI) we also have to evaluate the consequences of the meson cloud for semileptonic decays of octet baryons. Modifications of the axial-vector coupling constants due to the meson cloud can be quite important [17 19] since the corresponding axial-currents are not protected against renormalization due to the mesonic cloud.

The analysis of the EMC experiment under the assumption of $S U(3)$ symmetry (!) indicates that there may be a relation between the spin and strangeness content of the nucleon. The value for the polarized strange quark content of the nucleon obtained from such an analysis is $\Delta s=-0.19$ (newer SMC [15] and SLAC [16] experiments indicate a somewhat smaller amount), which suggests a revision of the simple view of the nucleon. In meson cloud models the strangeness content of the nucleon is generated by virtual fluctuations into strange mesons $\left(K\right.$ or $\left.K^{*}\right)$ and strange baryons $\left(\Lambda, \Sigma\right.$ or $\left.\Sigma^{*}\right)$. We stress the fact that the 'experimental' result obtained for the polarized strange quark content has been obtained under the assumption of $S U(3)$ symmetry, which is approximately realized in semileptonic decays [20]. On the other hand, $S U(3)$ symmetry is violated as far as the masses of hadrons are concerned and it is also violated in the sea quark distributions of the proton, which we discuss in the present paper. In models which include $S U(3)$ symmetry breaking effects the conclusion about the polarized strangeness content can be modified. On the other hand, the 
approximate validity of $S U(3)$ symmetry for the semileptonic decays of baryons has to be an important check for any baryonic model.

The discussion above shows that in order to understand the structure of the nucleon (the same is true for other baryons) one has to treat consistently the flavour and spin content. Because of the surprising success of the simple Cabibbo model, the semileptonic decays have to be treated simultaneously.

The pion, being the lightest meson was the first included in the deep-inelastic scattering [21,22]. It was suggested in Ref. [23] to include also other mesons which had proven to be important in the low-energy nucleon-nucleon and nucleon-hyperon scattering. Their effects in deep inelastic scattering are discussed in more detail in Ref. [10].

It has been suggested recently to study the virtual pion component in the nucleon in deep inelastic scattering experiments at HERA [24]. The semi-inclusive reaction $e p \rightarrow e^{\prime} n X$ is presently being studied by the ZEUS collaboration, which has installed a test forward neutron calorimeter to complement its leading proton spectrometer [25]. It is also worth mentioning that a recent lattice QCD calculations gave evidence for the importance of pion loop effects for nucleon properties [26]. The renewed interest in the virtual pions (mesons) in the nucleon, especially in connection to deep inelastic scattering, requires a better understanding of the methods used up to now in the literature on that subject.

In the meson cloud model the nucleon is viewed as a quark core, termed a bare nucleon, surrounded by the mesonic cloud. The convolution model seems to be the best tool to understand the structure of such a composed object. The internal consistency of the convolution model has been recently a subject of discussion [27,11]. A special emphasis has been put on satisfying sum rules [27, 11, 10]. When standard $t$-dependent form factors [27] are used, both number and momentum sum rules cannot be satisfied automatically. While number sum rules can be satisfied by independent adjustment of cut-off parameters of the vertex form factors for the diagrams with off-shell mesons and baryons, momentum conservation is violated to a degree dependent on the functional form of the form factor (see also [28]). It has been pointed out recently that special care is required, as far as form factors are 
concerned, in order to conserve both sum rules in a natural way 11$]$.

For the first time in the literature we present useful complete set of formulas for the helicity dependent splitting functions of the nucleon (octet baryon) into pseudoscalar/vector meson — octet/decuplet baryon states, i.e. we include all particles which turned out to be crucial in modern meson exchange models for nucleon-nucleon and nucleon-hyperon scattering at low energies 29,30. In addition to unpolarized deep-inelastic scattering we are also interested in possible effects of the meson cloud for the polarized deep-inelastic scattering. The proton helicity flow to the pionic cloud has been calculated recently in the relativistic light-cone perturbation theory including $N \pi N$ and $N \pi \Delta$ vertices [31]. The analysis of unpolarized deep-inelastic scattering suggests that also vector mesons $(\rho, \omega)$ may play an important role [10,32], provided rather hard form factors are used. The importance of vector mesons $(S=1)$ for deep inelastic scattering of polarized particles can be even greater. Therefore we extend the formalism of Ref. [31] to vector mesons. We derive useful formulas for both unpolarized and polarized deep-inelastic scattering.

In comparison to our earlier analysis [10], where only unpolarized deep-inelastic scattering has been considered, in the present paper we analyze possible effects of the meson cloud also for the polarized deep-inelastic scattering as well as for the semileptonic decays of the octet baryons. There are a few technical details in which the present analysis differs from the previous one [10]. In Ref. [10] we have assumed for simplicity a dipole form of the vertex form factors with an universal cut-off parameter for all mesons. The value of the cutoff parameters of the vertex form factors had been adjusted in Ref. [10 to reproduce the experimental data for $(\bar{u}(x)+\bar{d}(x)) / 2-\bar{s}(x)$. Since the universality of the dipole form factor is not obvious and the fit to the $(\bar{u}(x)+\bar{d}(x)) / 2-\bar{s}(x)$ data is not very sensitive to the value of the cut-off parameter in the present paper we take slightly different attitude. Following 33,11, we estimate the free parameters of our model by analyzing high-energy $p p \rightarrow n X$ and $p p \rightarrow \Delta^{++} X$ production processes in a one-boson-exchange model. In distinction to 111 we include also the effects of the vector meson Fock components. Then we first discuss various effects of the meson-baryon Fock components on the flavour structure of the nucleon 
and calculate the Gottfried Sum Rule. Next we present the results of calculations for the axial-vector coupling constants $g_{A}$ for all possible semileptonic decays of the octet baryons. Finally, we calculate the Ellis-Jaffe Sum Rule for the proton and neutron as well as the Bjorken Sum Rule.

\section{THE CONVOLUTION MODEL}

We expand the nucleon wave function in terms of a few principle Fock components. In this model the nucleon can be viewed as a bare nucleon (core), surrounded by a mesonic cloud. The wave function of the nucleon with helicity $+1 / 2$ can be schematically written as

$$
|N \uparrow\rangle=\sqrt{Z}\left[|N \uparrow\rangle_{b a r e}+\sum_{B M} \sum_{\lambda \lambda^{\prime}} \int d y d^{2} k_{\perp} \phi_{B M}^{\lambda \lambda^{\prime}}\left(y, k_{\perp}^{2}\right)\left|B^{\lambda}\left(y, \vec{k}_{\perp}\right) ; M^{\lambda^{\prime}}\left(1-y,-\vec{k}_{\perp}\right)\right\rangle\right]
$$

where $\sqrt{Z} \phi_{B M}^{\lambda \lambda^{\prime}}\left(y, k_{\perp}^{2}\right)$ is the probability amplitude that a physical nucleon with helicity $+1 / 2$ is in a state consisting of a virtual baryon $B$ with longitudinal momentum fraction $y$,

transverse momentum $\vec{k}_{\perp}$, and helicity $\lambda$, and a virtual meson $M$ with momentum fraction $1-y$, transverse momentum $-\vec{k}_{\perp}$, and helicity $\lambda^{\prime}$. Note, that the helicities $\lambda$ and $\lambda^{\prime}$ need not to add up to $+1 / 2$, since an additional relative angular momentum between the particles is possible. In Eq.(3) $Z$ is the standard wave function renormalization constant, which can be interpreted as the probability of the bare nucleon [34].

It can be expected, that the structure of the core is rather simple. Presumably, it can be described as a three quark system in the static limit. Of course, in the deep inelastic regime at higher $Q^{2}$ additional sea of perturbative nature is created unavoidably by the standard QCD evolution.

The main idea of the convolution approach is that there are no interactions among the particles in a multi-particle Fock state during the interaction with the hard photon in deep inelastic scattering (for a detailed discussion see [27]). This enables one to relate the contribution of a Fock state $B M$ to the nucleon structure function $F_{2}$, to the structure functions of either the struck meson $M$ or the struck baryon $B$ (see Fig. 1a,b) 


$$
\begin{aligned}
\delta_{M} F_{2}^{N}(x) & =\int_{x}^{1} d y f_{M B / N}(y) F_{2}^{M}\left(\frac{x}{y}\right) \\
\delta_{B} F_{2}^{N}(x) & =\int_{x}^{1} d y f_{B M / N}(y) F_{2}^{B}\left(\frac{x}{y}\right) .
\end{aligned}
$$

The convolution formulas can be written in an equivalent way in terms of quark distributions:

$$
q_{N}(x)=Z\left[q_{N, \text { bare }}(x)+\int_{x}^{1} f_{M B / N}(y) q_{M}\left(\frac{x}{y}\right) \frac{d y}{y}+\int_{x}^{1} f_{B M / N}(y) q_{B}\left(\frac{x}{y}\right) \frac{d y}{y}\right] .
$$

The main ingredients in the formulas above are the splitting functions $f_{M B / N}(y)$ and $f_{B M / N}(y)$, which are related to the probability amplitudes $\phi_{B M}$

$$
\begin{aligned}
& f_{B M / N}(y)=\int_{0}^{\infty} d k_{\perp}^{2} \sum_{\lambda \lambda^{\prime}}\left|\phi_{B M}^{\lambda \lambda^{\prime}}\left(y, k_{\perp}^{2}\right)\right|^{2} \\
& f_{M B / N}(y)=\int_{0}^{\infty} d k_{\perp}^{2} \sum_{\lambda \lambda^{\prime}}\left|\phi_{B M}^{\lambda \lambda^{\prime}}\left(1-y, k_{\perp}^{2}\right)\right|^{2} .
\end{aligned}
$$

Because the description of the nucleon as a sum of $M B$ Fock states is independent of the reaction mechanism, the relation

$$
f_{M B / N}(y)=f_{B M / N}(1-y)
$$

must hold. It simply expresses the fact, that if a meson $M$ carries a longitudinal momentum fraction $y$ of the nucleon momentum, the remaining part of the nucleon is a baryon with the remaining longitudinal momentum fraction $1-y$. Moreover this relation automatically ensures global charge conservation

$$
\left\langle f_{M B / N}\right\rangle=\left\langle f_{B M / N}\right\rangle
$$

and momentum conservation

$$
\left\langle x f_{M B / N}\right\rangle+\left\langle x f_{B M / N}\right\rangle=\left\langle f_{B M / N}\right\rangle
$$

where $\langle f\rangle$ and $\langle x f\rangle$ are the first and second moments of the splitting functions.

In this notation, the wave function renormalization constant is given as

$$
Z=\left[1+\sum_{M B}\left\langle f_{B M / N}\right\rangle\right]^{-1}
$$


Both the Ellis-Jaffe Sum Rule and the axial-vector coupling constants for semileptonic decays can be expressed with the help of matrix elements of the axial-currents $A_{\mu}^{a}=\bar{q} \gamma_{\mu} \gamma_{5}\left(\lambda^{a} / 2\right) q$ (see section VI), where $\lambda^{a}$ are the Gell-Mann matrices. The expansion (3) implies (see Fig. 2)

$$
\begin{aligned}
\left\langle N\left|A_{\mu}^{a}\right| N\right\rangle_{\text {dressed }}=Z\left(\left\langle N\left|A_{\mu}^{a}\right| N\right\rangle_{\text {bare }}\right. & +\sum_{B_{1} B_{2} M} \Delta f_{\left(B_{1} B_{2}\right) M / N}\left\langle B_{1}\left|A_{\mu}^{a}\right| B_{2}\right\rangle \\
& \left.+\sum_{M_{1} M_{2} B} \Delta f_{\left(M_{1} M_{2}\right) B / N}\left\langle M_{1}\left|A_{\mu}^{a}\right| M_{2}\right\rangle\right),
\end{aligned}
$$

where

$$
\begin{aligned}
\Delta f_{\left(B_{1} B_{2}\right) M / N} & =\sum_{\lambda \lambda^{\prime}} 2 \lambda \int_{0}^{1} d y \int_{0}^{\infty} d k_{\perp}^{2} \phi_{B_{1} M}^{\lambda \lambda^{\prime}}\left(y, k_{\perp}^{2}\right) \phi_{B_{2} M}^{* \lambda \lambda^{\prime}}\left(y, k_{\perp}^{2}\right) \\
\Delta f_{\left(M_{1} M_{2}\right) B / N} & =\sum_{\lambda \lambda^{\prime}} 2 \lambda^{\prime} \int_{0}^{1} d y \int_{0}^{\infty} d k_{\perp}^{2} \phi_{B M_{1}}^{\lambda \lambda^{\prime}}\left(1-y, k_{\perp}^{2}\right) \phi_{B M_{2}}^{* \lambda \lambda^{\prime}}\left(1-y, k_{\perp}^{2}\right) .
\end{aligned}
$$

The essential ingredients in our model are the amplitudes $\phi_{B M}^{\lambda \lambda^{\prime}}\left(y, k_{\perp}^{2}\right)$. To calculate these quantities we employ the time-ordered perturbation theory (TOPT) 35,36,34 which has the advantage that the intermediate Fock states can be written down explicitly:

$$
\phi_{B M}^{\lambda \lambda^{\prime}}(\vec{p}, \vec{k}, \vec{q}=\vec{p}-\vec{k})=N_{N} \frac{N_{B}}{(2 \pi)^{\frac{3}{2}}} \frac{N_{M}}{(2 \pi)^{\frac{3}{2}}} \frac{V\left(\vec{p}, \uparrow ; \vec{k}, \lambda ; \vec{q}, \lambda^{\prime}\right)}{E_{N}-E_{M}-E_{B}}
$$

(compare [34]). This formula gives the amplitude of finding a nucleon with momentum $\vec{p}$ and helicity $+1 / 2$ in a Fock state where the baryon $B$ has the momentum $\vec{k}$, helicity $\lambda$ and the meson $M$ the momentum $\vec{q}=\vec{p}-\vec{k}$ and helicity $\lambda^{\prime}$. The factors $N_{N}\left(N_{B}\right)$ are the usual fermion wave function normalization factors $N_{N}=\sqrt{m_{N} / E_{N}}, N_{B}=\sqrt{m_{B} / E_{B}} ; N_{M}$ is a bosonic normalization factor $N_{M}=1 / \sqrt{2 E_{M}}$. The important feature of TOPT is, in contrast to the covariant perturbation theory, that the intermediate particles are on their mass-shell. Therefore, the vertex function $V$ in Eq.(16) can be calculated by using on-mass shell spinors. $V$ depends on a particular model, i.e. on the form of the lagrangian used. In general $V$ can be written as

$$
V(\vec{p}, \vec{k}, \vec{q})=\bar{u}_{N}(\vec{p})_{\alpha} v^{\alpha \beta \gamma} \chi_{\beta}(\vec{q}) \psi_{\gamma}(\vec{k})
$$

where summing and averaging over all possible spin-states is implicitly assumed. $\alpha, \beta$ and $\gamma$ are bi-spinor and/or vector indices dependent on the representation used for particles of a 
given type. $\chi$ and $\psi$ are the wave functions (field operators) of the intermediate meson and baryon, respectively.

It has been shown [34] for the $\pi N$ case, that in the infinite momentum frame (IMF) contributions of Fock states with anti-particles vanish and only contributions with forward moving particles survive. This statement is also true for other Fock states we are dealing with. In the IMF-limit the momenta of the particles involved can be parameterized in terms of $y$ and $\vec{k}_{\perp}$ :

$$
\vec{k}=y \vec{p}+\vec{k}_{\perp}, \quad \vec{k}_{\perp} \cdot \vec{p}=0, \quad \vec{q}=(1-y) \vec{p}-\vec{k}_{\perp} .
$$

In the limit $p=|\vec{p}| \rightarrow \infty$ only states with $y \in[0,1]$ do not vanish [34], and the amplitudes $\phi_{B M}$ can be expressed as

$$
\phi_{B M}\left(y, k_{\perp}^{2}\right)=\frac{1}{2 \pi \sqrt{y(1-y)}} \frac{\sqrt{m_{N} m_{B}} V_{I M F}\left(y, k_{\perp}^{2}\right)}{m_{N}^{2}-M_{B M}^{2}\left(y, k_{\perp}^{2}\right)}
$$

with $M_{B M}^{2}$ being the invariant mass squared of the $B M$ Fock state

$$
M_{B M}^{2}\left(y, k_{\perp}^{2}\right)=\frac{m_{B}^{2}+k_{\perp}^{2}}{y}+\frac{m_{M}^{2}+k_{\perp}^{2}}{1-y} .
$$

$V_{I M F}$ is the vertex-function in the IMF. In the formula above an extra factor $(\pi p)^{-1 / 2}$ has been taken out. It would cancel when going to probability densities by an appropriate factor of the jacobian of the transformation (18).

If the vertex function used does not contain a derivative of the meson field, we could have equally well used covariant perturbation theory instead of TOPT. But if the vertex function used contains a derivative of the meson field this is no longer true. To illustrate this point let us consider the example of the pseudovector $N \pi N$ vertex, given by

$$
\mathcal{L}_{p v}=\bar{u} \gamma_{5} \gamma^{\mu} \partial_{\mu} \pi u
$$

where for simplicity the coupling constant and isospin structure have been suppressed. The standard covariant technique [11,22] leads to the following splitting function of the meson 


$$
\begin{aligned}
f_{M B / N}^{p v}(y)= & \frac{1}{16 \pi^{2}} \frac{1}{(1-y)^{2} y} \int_{0}^{\infty} d k_{\perp}^{2}\left|G\left(1-y, k_{\perp}^{2}\right)\right|^{2} \\
& \cdot \frac{\left(m_{B}+m_{N}\right)^{2}\left[\left(m_{N}(1-y)-m_{B}\right)^{2}+k_{\perp}^{2}\right]}{\left[m_{N}^{2}-M_{B M}^{2}\left(1-y, k_{\perp}^{2}\right)\right]^{2}} .
\end{aligned}
$$

The result for the baryon is:

$$
\begin{aligned}
f_{B M / N}^{p v}(y)= & \frac{1}{16 \pi^{2}} \frac{1}{(1-y) y^{2}} \int_{0}^{\infty} d k_{\perp}^{2}\left|G\left(y, k_{\perp}^{2}\right)\right|^{2} \\
& \left(m_{N}^{2} m_{B}^{2}(1-y)^{2}+k_{\perp}^{2}\left(m_{B}+m_{N}\right)^{2}-2 m_{N} m_{B}\left(y m_{M}^{2}+k_{\perp}^{2}\right)\right. \\
& \left.+\frac{1}{(1-y)^{2}}\left(y m_{M}+k_{\perp}^{2}\right)^{2}\right) \frac{1}{\left[m_{N}^{2}-M_{B M}^{2}\left(y, k_{\perp}^{2}\right)\right]^{2}}
\end{aligned}
$$

Here the two results are not related by Eq.(9), which leads to violation of the charge- and momentum conservation. The reason for this puzzle is, that by using a derivative coupling, an additional off-shell dependence is introduced into the vertex function, which cannot be suppressed in the IMF-limit. A way out is to use TOPT. Here, however, the problem arises how to choose the meson energy in the vertex. In principle, there are two possible prescriptions:

A) One uses in the vertex the meson four-momentum $q^{\mu}: \bar{u}_{N} \gamma_{5} \gamma_{\mu}(-i) q^{\mu} u_{B}$, i.e. the meson energy in the vertex is $E_{M}$. With this form of the vertex one reproduces the baryon splitting function given by Eq.(23). The meson splitting function is related to this result by Eq.(9).

B) Instead of $q^{\mu}$ one uses the difference of the baryon four-momenta $p^{\mu}-k^{\mu}$ : $\bar{u}_{N}(p) \gamma_{5} \gamma_{\mu}(-i)(p-k)^{\mu} u_{B}(k)$, i.e. the meson energy in the vertex is $E_{N}-E_{B}$. With this prescription one gets the splitting function given by Eq.(22). Also here the resulting splitting functions for the baryon and meson fulfill Eq.(9).

Thus TOPT, in contrast to a covariant calculation, is consistent with the convolution approach. The remaining point to clarify is which of the two prescription one should use. We will always use prescription $\mathrm{B}$, because in this prescription the splitting functions for the pseudovector case are identical to those of the pseudoscalar case, if the coupling constants 
are identified properly. Moreover, in this prescription the structure of the vertex is due to the baryonic current only [37].

\section{FORM FACTORS}

The most general structure of the hadronic tensor must include the dependence on offshell effects 38]. Because a realistic calculation of such off-shell effects is not possible at present, we shall neglect such effects in this paper. Because of the extended nature of the hadrons involved one has to introduce phenomenological vertex form factors, which parameterize complicated unknown microscopic effects. The form factors are included in the calculation by replacing the vertex function $V\left(y, k_{\perp}^{2}\right)$ by $V^{\prime}\left(y, k_{\perp}^{2}\right)=G\left(y, k_{\perp}^{2}\right) V\left(y, k_{\perp}^{2}\right)$.

Now relation (9), which can be shown to hold exactly in the case of point-like particles (in either covariant and TOPT calculations), imposes a severe restriction to these form factors:

$$
G_{B M}\left(y, k_{\perp}^{2}\right)=G_{M B}\left(1-y, k_{\perp}^{2}\right)
$$

The form factors often used in meson exchange models and convolution models are functions of $t$ only, the four-momentum squared of the meson. It is to be expected that form factors which depend only on the kinematical variables of only one of the two particles of the two-body system, like the often used dipole form factor

$$
G(t)=\left(\frac{\Lambda^{2}-m_{M}^{2}}{\Lambda^{2}-t}\right)^{2},
$$

will not satisfy Eq.(9). It can be shown 27] that these form factors do not conserve basic quantities like charge and momentum simultaneously. One simple method to obtain form factors with the right symmetry is to multiply a $t$-dependent form factor by a $u$-dependent one with the same functional form with $m_{M}$ replaced by $m_{B}$

$$
G_{\text {sym }}(t, u)=G\left(t, m_{M}\right) G\left(u, m_{B}\right) .
$$

In terms of the IMF variables $y$ and $k_{\perp}^{2}, t$ and $u$, the four momentum squared of the intermediate baryon, is given by 


$$
\begin{aligned}
& t=-\frac{k_{\perp}^{2}}{y}-(1-y)\left(\frac{m_{B}^{2}}{y}-m_{N}^{2}\right) \\
& u=-\frac{k_{\perp}^{2}}{1-y}-y\left(\frac{m_{M}^{2}}{1-y}-m_{N}^{2}\right) .
\end{aligned}
$$

The importance of using such symmetric form factors was noticed only recently 11$]$. Another possible approach, to fix the cut-off parameters to assure number sum rules (global charge conservation) (see [27,28]) is in our opinion somewhat arbitrary, and does not guarantee momentum conservation.

In numerical calculations we use vertex form factors in the exponential form

$$
G_{B M}\left(y, k_{\perp}^{2}\right)=\exp \left[\frac{m_{N}^{2}-M_{B M}^{2}\left(y, k_{\perp}^{2}\right)}{2 \Lambda^{2}}\right]
$$

where $M_{B M}^{2}\left(y, k_{\perp}^{2}\right)$ is the invariant mass squared of the baryon-meson $B M$ Fock state

$$
M_{B M}^{2}\left(y, k_{\perp}^{2}\right)=\frac{m_{B}^{2}+k_{\perp}^{2}}{y}+\frac{m_{M}^{2}+k_{\perp}^{2}}{1-y} .
$$

A form factor of this type, first introduced in Ref. [11], fulfills the necessary symmetry conditions Eq. (9). Parameterization of the vertex form factors in terms of the invariant mass of the two-body system is very natural in the light-cone approach. Furthermore, the light-cone approach is the best suited for applications to deep inelastic scattering.

In order to fix the cut-off parameters $\Lambda$ of the form factors we use high-energy baryon production data. The neutron production data in the $p p \rightarrow n X$ scattering seems to be best tailored for extracting the cut-off parameter for the $N \pi$ and $N \rho$ Fock states. Because we shall limit ourselves to data for relatively low exchanged four-momenta, it is reasonable to assume that the neutron is produced by a simple one-boson-exchange mechanism (OBE) (see Fig.3). In this model the differential cross section can be described as a product of the probability for a proton being in a $n \pi^{+}$or $n \rho^{+}$Fock state and the total cross section of $\pi^{+}\left(\rho^{+}\right) p$ scattering. In general, the invariant cross section for $p p \rightarrow B X$ production in the OBE model is

$$
E \frac{d^{3} \sigma(p p \rightarrow B X)}{d^{3} p}=\frac{y}{\pi} \frac{d^{2} \sigma}{d y d k_{\perp}^{2}}=\frac{y}{\pi} \sum_{\lambda \lambda^{\prime}}\left|\phi_{B M}^{\lambda \lambda^{\prime}}\left(y, k_{\perp}^{2}\right)\right|^{2} \cdot \sigma_{t o t}^{M p}(s(1-y))
$$


Here $y$ is the longitudinal momentum fraction of the baryon with respect to the momentum of the incoming proton; $\vec{k}_{\perp}$ the corresponding transverse momentum. Specialized to neutron production with $\pi^{+}$-exchange this formula reads:

$$
\begin{aligned}
E \frac{d^{3} \sigma(p p \rightarrow n X)}{d^{3} p}= & \frac{g_{p n \pi^{+}}^{2}}{16 \pi^{3}} \frac{(1-y)^{2} m_{N}^{2}+k_{\perp}^{2}}{\left[m_{N}^{2}-M_{N \pi}^{2}\left(y, k_{\perp}^{2}\right)\right]^{2}} \frac{\left|G_{N N \pi}\left(y, k_{\perp}^{2}\right)\right|^{2}}{y(1-y)} \\
& \cdot \sigma_{t o t}^{\pi p}(s(1-y)) .
\end{aligned}
$$

For $\rho^{+}$-exchange the formula is much longer and will be not presented here. The necessary ingredients can be found in Appendix B.

These formulas are strictly valid only in the IMF-limit, but for a sufficiently high energy they are a good approximation. In the following we neglect the slow energy dependence of the total $\pi N$ cross section and use a value of $\sigma_{\text {tot }}^{\pi^{+} p}=23.8 \pm 0.1 \mathrm{mb}$ [39]. In addition, due to the lack of experimental data we also assume $\sigma_{t o t}^{\rho^{+} p}=\sigma_{t o t}^{\pi^{+} p}$.

In Fig. 4 we show a fit by the OBE model to the experimental data (the $k_{\perp}^{2}=0$ data are taken from Ref. [40] with $\sqrt{s}=53 \mathrm{GeV}$; the other data are taken from Ref. [41] with $p_{L A B}=24 \mathrm{GeV} / \mathrm{c}$ ). In this calculation we take $g_{p p \pi^{0}}^{2} / 4 \pi=13.6 \pm 0.1$ [42] and $g_{p p \rho^{0}}^{2} / 4 \pi=0.84$, $f_{p p \rho^{0}} / g_{p p \rho^{0}}=6.1$ [30]. As a criterion for the fit we have assumed that the calculated result must not exceed the experimental data. For low $k_{\perp}^{2} \pi$-exchange is the dominant contribution, the $\rho$-exchange plays a rather marginal role. For higher $k_{\perp}^{2} \rho$-exchange becomes the dominant mechanism. Thus the cut-off parameter for the $N N \pi$ and $N N \rho$ vertices can be fixed almost unambiguously. The fit to the experimental data yields $\Lambda_{N N \pi}=\Lambda_{N N \rho}=1.10 \pm 0.05 \mathrm{GeV}$.

We obtain a good description of the experimental data at intermediate $y=0.4-0.9$. Outside this region the fit fails. This is to be expected, because for low $y$ one could expect additional contributions from e.g. multi-meson exchange processes. For our purpose (later we shall concentrate on moments of $x$-distributions rather than the distributions themselves) the assumption of a OBE mechanism is sufficient, as the maximum cross section lies at intermediate $y$, where the mesons can be treated as not reggeized. The correct $k_{\perp}^{2}$-dependence obtained from the fit is a further argument in favor of the simple OBE model. Despite the success of the fit one should bear in mind that we obtain only an upper bound of the 
$\rho$-exchange contribution.

In order to extract information about the $N K \Lambda$ vertex, one can examine high-energy $\Lambda$ production in the $p p \rightarrow \Lambda X$ reaction in the same way as neutron production. Also here we assume that the dominant mechanism is a OBE mechanism, $K$ or $K^{*}$-exchange. The differential cross section for $K$-exchange is given by

$$
\begin{aligned}
E \frac{d^{3} \sigma(p p \rightarrow \Lambda X)}{d^{3} p}= & \frac{g_{p \Lambda K^{+}}^{2}}{16 \pi^{3}} \frac{\left(y m_{N}-m_{\Lambda}\right)^{2}+k_{\perp}^{2}}{\left[m_{N}^{2}-M_{\Lambda K}^{2}\left(y, k_{\perp}^{2}\right)\right]^{2}} \frac{\left|G_{N \Lambda K}\left(y, k_{\perp}^{2}\right)\right|^{2}}{y(1-y)} \\
& \cdot \sigma_{\text {tot }}^{K p}(s(1-y)) .
\end{aligned}
$$

For $K^{*}$-exchange we refer to Eq. (31) and Appendix B.

We have taken $g_{p \Lambda K^{+}}^{2} / 4 \pi=14.7$, which is related by $S U(6)$ symmetry to the $g_{p p \pi^{0}}^{2}$ given before. The coupling constant obtained from experiment is $15.4 \pm 1.5$ [43] which supports the assumption of $S U(6)$ symmetry. Also for the coupling constants involving vector mesons we assume $S U(6)$ symmetry (for details see [30]). The total cross section $\sigma_{\text {tot }}^{K^{+} p}$ is $19.9 \pm 0.1 \mathrm{mb}$ [39]. For $\sigma_{t o t}^{K^{*+} p}$ we assume the same value. The fit of the cut-off parameters $\Lambda_{N \Lambda K}$ and $\Lambda_{N \Lambda K^{*}}$ is shown in Fig.4. The general features of the fit are similar to those found for neutron production. Also, the cut-off parameters $\Lambda_{N \Lambda K}=\Lambda_{N \Lambda K^{*}}=1.05 \pm 0.05 \mathrm{GeV}$ obtained from the fit are quite similar to those found for $\pi$ - and $\rho$-exchange. Thus, it is possible to assume a kind of universality and use a cut-off parameter $\Lambda=1.08 \mathrm{GeV}$ for all vertices involving octet baryons and pseudoscalar or vector mesons. This is rather attractive, that the same cut-off parameters can be used for pseudoscalar and vector mesons.

In the literature, instead of the symmetric form factor given by Eq.(29), a dipole form factor Eq. (25) is often used. As discussed above, the use of such form factors leads to a violation of basic symmetries. In Fig.5 we show, for example, the differential cross section for neutron production calculated using the dipole form factor $(\Lambda=1.2 \mathrm{GeV}[10])$. As seen from the figure, one gets a satisfactory description for small $k_{\perp}^{2}$. One cannot, however, describe the data at large $k_{\perp}^{2}$. Comparison of Fig.4 and Fig.5 clearly indicates a preference for the symmetric form factor (Eq. 29).

The violation of the Gottfried Sum Rule is an interplay between the $\pi N$ and $\pi \Delta$ com- 
ponents and crucially depends on vertex form factors, i.e. its functional form and cut-off parameter. For instance, taking the dipole form and assuming the same cut-off for both components leads to strong cancellations. As a consequence only about half of the experimentally observed violation can be explained [10,44, 12]. In order to shed light on the $N \pi \Delta$ vertex we follow [33] and examine $\Delta^{++}$production in $p p$ and $p \bar{p}$ collisions. The differential cross section for one-pion-exchange (Fig.6) is given by:

$$
\begin{aligned}
E \frac{d^{3} \sigma\left(h p \rightarrow \Delta^{++} X\right)}{d^{3} p}= & \frac{g_{p \Delta^{++} \pi^{-}}^{2}}{16 \pi^{3}} \frac{\left|G_{N \Delta \pi}\left(y, k_{\perp}^{2}\right)\right|^{2}}{y(1-y)} \\
& \cdot \frac{\left[\left(y m_{N}+m_{\Delta}\right)^{2}+k_{\perp}^{2}\right]^{2}\left[\left(y m_{N}-m_{\Delta}\right)^{2}+k_{\perp}^{2}\right]}{6 y^{2} m_{\Delta}^{2}\left[m_{N}^{2}-M_{\Delta \pi}^{2}\left(y, k_{\perp}^{2}\right)\right]^{2}} \\
& \cdot \sigma_{\text {tot }}^{\pi h}(s(1-y)) .
\end{aligned}
$$

The differential cross section for $\rho$-exchange can be calculated analogously (see Eq. 31 and Appendix B).

As coupling constants we take $f_{p \Delta^{++} \pi^{-}}^{2} / 4 \pi=12.3 \mathrm{GeV}^{-2}$ and $f_{p \Delta^{++} \rho^{-}}^{2} / 4 \pi=34.7 \mathrm{GeV}^{-2}$ [30]. The available $\Delta^{++}$-production spectra [45] are biased by an ambiguity in extraction of background contributions. Thus, in Fig.7 two scales are shown (different background assumptions). The experimental data shown in Fig.7 have been taken at three different energies $\sqrt{s}=100,200$ and $360 \mathrm{GeV}$. We do not distinguish the data taken at different energies, as no energy dependence is visible. This fact is in agreement with the simple assumption of an OBE mechanism and supports the IMF-approach. As far as the absolute normalization is concerned some extra experimental information is needed. We use more precise $y$-integrated-spectra dependent only on $k_{\perp}^{2}$ 45,46 for this purpose. The result of the fit shown in Fig.7 and Fig.8 yields $\Lambda_{N \Delta \pi}=\Lambda_{N \Delta \rho}=0.98 \pm 0.05 \mathrm{GeV}$. In contrast to the $n$ and $\Lambda$ production an unambiguous distinction between the $\pi$ and $\rho$ exchange contributions is not possible. For simplicity, we have assumed the same cut-off parameters for both, in analogy to the octet baryon production.

We assume that the cut-off parameters for the whole decuplet to be the same as those obtained from the $p p \rightarrow \Delta^{++} X$ production process. This universality within an $S U(3)$ multiplet greatly reduces the number of, in principle unknown, parameters. 
To reduce the number of parameters further, we employ $S U(6)$ symmetry as mentioned above to relate coupling constants. Thus only the coupling of the $\omega$ to the nucleon remains to be fixed. It has to be treated differently, because the $\omega$ is an almost ideal mixture of an octet and a singlet [30]

$$
\omega=\frac{1}{\sqrt{3}} \omega_{8}+\sqrt{\frac{2}{3}} \omega_{1} .
$$

The $\omega$-couplings were found to be: $g_{p p \omega}^{2} / 4 \pi=8.1$ and $f_{p p \omega} / g_{p p \omega}=0$ [30].

It is interesting to ask the question of how sensitive is our model to the functional form of the form factor. In the following we restrict ourselves to form factors which have the correct symmetry. The simplest are the symmetric monopole $(n=1)$ or dipole $(n=2)$ form factors:

$$
G_{n}(t, u)=\left(\frac{\Lambda^{2}-m_{M}^{2}}{\Lambda^{2}-t}\right)^{n}\left(\frac{\Lambda^{2}-m_{B}^{2}}{\Lambda^{2}-u}\right)^{n} .
$$

In principle, one might expect that they produce results different from the exponential form (Eq. 29) for the first moments of the splitting functions, because they cut off the integrands much more softly. Surprisingly, this is not the case. In Table 1 we show values of cutoff parameters of the exponential, monopole and dipole form factors fitted to the neutron production data and the corresponding moments of the splitting functions. Almost the same first moments are obtained with the different functional forms. Our results are almost insensitive to the form of the form factors used; it is rather the symmetry (Eq. 9) which allows one to describe the data in a broad range of $y$ and $k_{\perp}^{2}$.

\section{MESON CLOUD EFFECTS ON THE FLAVOUR STRUCTURE OF THE NUCLEON}

The renewed interest [47,44,12,48,23,10] in the meson cloud of the nucleon comes from the fact that it provides a natural explanation of the $\bar{d}-\bar{u}$ asymmetry and, as a consequence, the violation of the Gottfried Sum Rule. The Gottfried Sum Rule can be expressed as 


$$
S_{G}=\int_{0}^{1}\left[F_{2}^{p}(x)-F_{2}^{n}(x)\right] \frac{d x}{x}=\frac{1}{3} \int[u(x)+\bar{u}(x)-d(x)-\bar{d}(x)] d x=\frac{1}{3}+\frac{2}{3} A_{\bar{u}-\bar{d}}
$$

where $q(\bar{q})$ are the light quark (antiquark) distributions in the proton and $A_{\bar{u}-\bar{d}}=$ $\int_{0}^{1}[\bar{u}(x)-\bar{d}(x)] d x$. In obtaining Eq.(37) isospin symmetry within the nucleon doublet has been assumed]. In accordance with the simple structure of the bare nucleon, it is natural to expect that the only sea in the bare nucleon is of perturbative origin. Such a sea, being $S U(2)$ symmetric, does not contribute to $A_{\bar{u}-\bar{d}}$. In our model the asymmetry is caused by the valence quarks in the virtual mesons. Only $\pi$ and $\rho$ mesons contribute to the asymmetry [10], which can be expressed in terms of the first moments of the corresponding splitting functions

$$
A_{\bar{u}-\bar{d}}=Z\left[-\left\langle f_{\pi^{+} n / p}\right\rangle+\left\langle f_{\pi^{-} \Delta^{++} / p}\right\rangle-\left\langle f_{\pi^{+} \Delta^{0} / p}\right\rangle-\left\langle f_{\rho^{+} n / p}\right\rangle+\left\langle f_{\rho^{-} \Delta^{++} / p}\right\rangle-\left\langle f_{\rho^{+} \Delta^{0} / p}\right\rangle\right] .
$$

Contributions of a perturbative nature as well as any symmetric sea in mesons and/or baryons would not change this result, because $S_{G}$ is sensitive only to the difference $\bar{u}-\bar{d}$ (Eq. 37). In Table 2 we show various contributions to the $\bar{u}-\bar{d}$ asymmetry and to the Gottfried Sum Rule. The inclusion of the $\pi N$ Fock component leads to a reduction of $S_{G}$ from $1 / 3$ to 0.230 . In comparison to earlier works where an universal cut-off parameter of the dipole form factor has been assumed for simplicity [12,10], the procedure proposed in section III leads to important modifications. We find a much smaller probability of the $\pi \Delta$ (6.2\% in comparison to $16.1 \%$ in Ref. [10]) Fock component and a substantially larger probability of the $\rho N$ component (10.9\% in comparison to only $1.6 \%$ in Ref. [10]). Summarizing, including all components in the nucleon wave function expansion (3) leads to a good agreement with the NMC result [1] of the Gottfried Sum Rule $S_{G}=0.24 \pm 0.016$ (see Table 2). It should be noted here, that the 'true' experimental value of $S_{G}$ might be even somewhat smaller than the NMC result due to shadowing effects in the deuteron [11,50].

\footnotetext{
${ }^{1}$ The violation of the isospin symmetry of proton and neutron could in principle be another important effect 49]. Up to now no quantitative estimate of this effect exists.
} 
In calculating the $\bar{u}(x)-\bar{d}(x)$ asymmetry in the present paper we have neglected the interference diagrams like $\pi^{0}-\eta$ or $\rho-\omega$. While the contributions of such diagrams to the number and momentum sum rule cancel, their contribution to $\bar{u}(x), \bar{d}(x)$ as well as to the $\bar{u}(x)-\bar{d}(x)$ asymmetry is not protected by any conserved current and is in principle possible. A reliable calculation of the $x$-dependence of the $\bar{u}-\bar{d}$ difference is rather difficult and requires microscopic models for the mesons involved. In contrast to the $x$-dependence the bulk effect of $\bar{u}-\bar{d}$ can be easily estimated assuming that the discussed mesons are members of the $S U(3)$ multiplets. The contribution of the interference diagrams of two mesons to the $\bar{u}-\bar{d}$ difference can be calculated in a full analogy to the diagonal terms:

$$
\langle\bar{u}-\bar{d}\rangle=\int_{0}^{1} d y \int d k_{\perp}^{2} \sum_{\lambda \lambda^{\prime}} \phi_{B M_{1}}^{\lambda \lambda^{\prime}}\left(y, k_{\perp}^{2}\right) \phi_{B M_{2}}^{\lambda \lambda^{\prime}, *}\left(y, k_{\perp}^{2}\right)\left\langle M_{2}|\bar{u}-\bar{d}| M_{1}\right\rangle,
$$

where the matrix element $\left\langle M_{2}|\bar{u}-\bar{d}| M_{1}\right\rangle$ can be calculated using the $S U(6)$ wave functions of the mesons involved. We find $\langle\bar{u}-\bar{d}\rangle=0.0050$ and -0.0011 for $\pi^{0}-\eta$ and $\rho-\omega$, respectively. These contributions have to be compared to $-0.12(\pi)$ and $-0.073(\rho)$ obtained from the diagonal mesonic terms. There are different reasons for the smallness of these contributions. While the $\pi^{0}-\eta$ is small due to a small coupling constant $g_{N N \eta}$, the $\rho-\omega$ contribution is small due to the vanishing tensor coupling constant $f_{N N \omega}$. Thus the peculiarities of the nucleon-meson-nucleon couplings allows one to safely neglect the interference terms.

As has been emphasized in Ref. [23], an attractive feature of the meson cloud is that a large fraction of the nucleon sea can be attributed to the virtual mesons (see also Ref. [22]). Let us consider this issue in more detail in the case of the present model. For this purpose the global CCFR experimental data [51]

$$
\begin{aligned}
\kappa & =\frac{2\langle x s\rangle}{\langle x \bar{u}\rangle+\langle x \bar{d}\rangle} \quad=0.44_{-0.07-0.02}^{+0.09+0.07}, \\
\eta_{s} & =\frac{2\langle x s\rangle}{\langle x u\rangle+\langle x d\rangle} \quad=0.057_{-0.008-0.002}^{+0.010+0.007}, \\
R_{Q} & =\frac{\langle x \bar{u}\rangle+\langle x \bar{d}\rangle+\langle x \bar{s}\rangle}{\langle x u\rangle+\langle x d\rangle+\langle x s\rangle}=0.153 \pm 0.034
\end{aligned}
$$

obtained at an average value of $Q^{2}=16.85(\mathrm{GeV} / \mathrm{c})^{2}$ are useful. In the formulas above $\langle x q\rangle$ denote second moments of the quark distributions $q(x)$. Obtaining the quantities given by 
Eq.(40) in our model is not straightforward. First of all, the experimental data has been measured at relatively large $Q^{2}$. Therefore, the model has to be extended by the inclusion of extra sea of a perturbative nature. Secondly, the quantities in Eq.(40) are sensitive to the valence quark distributions which cannot be calculated fully consistently [10]. In spite of these difficulties it seems instructive to make an estimate of those quantities. It is consistent within our approximation scheme to assume a simple structure of bare baryons and take into account fully dressed mesons.

Calculation of the CCFR quantities implies knowledge of the $x$-dependences of the quark distributions. For the mesons we take quark distribution functions known from the analysis of the Drell-Yan processes in the pion-nucleus collisions 52,53. For the bare baryons the situation is somewhat more complicated, and some approximations are unavoidable. For the purpose of comparison with the global quantities like those given by Eq.(40) it is sufficient to approximate valence quark distributions by those of the physical baryons. A similar approximation for the sea quarks is, however, not realistic as within our model a large fraction of the nucleon's sea is included explicitly via the pionic (mesonic) cloud. As discussed in Ref. [7], this effect can be approximately included by reducing the total nucleon's sea, known from DIS, by a factor $R_{\text {sea }}$.

Our model, as discussed later, predicts both asymmetry between $\bar{u}$ and $\bar{d}$ quark distributions and a suppression of the strange sea in comparison to the non-strange sea. On the other hand, perturbative QCD predicts $\bar{u}-\bar{d}$ symmetry and allows for relative suppression of the strange component. It is an empirical fact that at $Q^{2}=4 \mathrm{GeV}^{2}$ the strange sea is suppressed by a factor 2. It is interesting how much of this effect is of perturbative/nonperturbative nature. One could use the CCFR parameter $\kappa$ (see Eq.(40)) to fix the strangeness suppression factor $R_{s t r}$ for the sea quark distributions in mesons, baryons and the bare nucleon (not dressed with mesons),

$$
s_{\text {sea }}\left(x, Q^{2}\right)=\bar{s}_{\text {sea }}\left(x, Q^{2}\right)=R_{\text {str }} \bar{u}_{\text {sea }}\left(x, Q^{2}\right)=R_{\text {str }} \bar{d}_{\text {sea }}\left(x, Q^{2}\right) .
$$

In the present paper we adjust the two unknown parameters $R_{\text {sea }}$ and $R_{\text {str }}$ to the CCFR 
quark distributions $\bar{q}(x)=\bar{u}(x)+\bar{d}(x)+\bar{s}(x)$ [54 and to the CCFR parameter $\kappa$ [55]. In principle both $\bar{q}(x)$ and $\kappa$ depend explicitly on $R_{\text {sea }}, R_{s t r}$. In practice the situation is simpler because $\bar{q}(x)$ depends mainly on $R_{\text {sea }}$ and is almost independent of $R_{\text {str }}$ while $\kappa$ depends mainly on $R_{\text {str }}$ and is almost independent of $R_{\text {sea }}$. This allows us to extract the two parameters. If we take the $S_{0}^{\prime}$ MSR [3] sea parameterization for the bare nucleon (we assume that the valence quark distributions in baryons are related via $S U(3)$ symmetry), we find $R_{\text {sea }}=0.4$ and $R_{\text {str }}=0.75$. The corresponding $\bar{q}(x)$ is compared with the "experimental" sea quark distribution of the CCFR collaboration [54] in Fig.9 and the resulting parameter $\kappa$ $=0.40$. The other CCFR parameters are then: $\eta_{s}=0.069, R_{Q}=0.201$, slightly above their experimental counterparts. The small overestimation can be easily understood because these two quantities are sensitive to the valence quark distributions which cannot be calculated fully consistently [10]. We expect the values of $R_{Q}$ and $\eta_{s}$ to be biased by the approximations made up to $15 \%$.

While $R_{\text {sea }}$ depends to some extend on the parameterization of the sea quark distributions used, $R_{\text {str }}$ is fairly stable. Therefore the result of the fit suggests that the empirical suppression of the perturbative strange sea component at $Q^{2}$ values of a few $\mathrm{GeV}^{2}$ is about 0.75 in comparison to the total strange sea suppression of 0.5. The difference is due to nonperturbative effects of the meson cloud.

Not only global quantities like the Gottfried Sum Rule or the CCFR parameters are of interest. Also the $x$-dependence of various quantities is helpful in testing models. It is known that sufficiently far above the strangeness production threshold the QCD evolution equations [56] lead to the production of a perturbative $S U(3)$ symmetric sea. In this context it is useful to study differences of quark distributions instead of the distributions themselves, especially those describing the $x$-dependence of the $S U(2)$ and $S U(3)$ symmetry violation of the sea quarks [10,7].

The $x(\bar{d}(x)-\bar{u}(x))$ is the quantity which describes the $x$-dependence of the $S U(2)$ symmetry breaking of the nucleon sea. This quantity cannot be easily obtained from the integrand of $S_{G}$ which, in addition to $\bar{d}-\bar{u}$, involves the $x$-dependence of valence quark distributions 
(see Eq.37), making the extraction of the interesting quantity rather difficult. The analysis of dilepton production in $p p$ and $p d$ collisions seems to be much better in this respect [6.7. $x(\bar{d}-\bar{u})$ is of special importance since it can be used to verify our model as it does not require further parameters. Having fixed the cut-off parameters of the vertex form factors from hadronic reactions it can be obtained parameter free. If one assumes that the sea of the bare nucleon is $S U(2)$ symmetric, then only pions (mesons) contribute to $x(\bar{d}-\bar{u})$. If, in addition, one assumes that the pion sea is also $S U(2)$ symmetric (there are no reasons for an asymmetry), then the whole effect comes from the valence quarks in the pion which are relatively well known from the Drell-Yan processes. In Fig. 10a we show the predictions of our model for three different pion structure functions: the leading order (LO) parameterization of the NA3 collaboration 52] (dotted line), the next-to-leading order (NLO) parameterization of Ref. [53] (solid line) and structure function of the pion calculated in a model of the radiatively generated sea [57] (dashed line). As seen from the figure the results for different parameterizations are rather similar. In Fig. 10b we compare our result (solid line) with parameterizations of the quark distributions which have been fitted to the world data on DIS and Drell-Yan processes. It is worth noting that our result is almost identical for $x<0.2$ with a recent NLO parameterization MSR(A) of the MSR group [4] which includes both the new HERA data [58,59] and the experimental result of the Drell-Yan NA51 experiment [5]. The Drell-Yan experiment planned at Fermilab [6] will be a severe test of our model and should shed new light on the origin of the Gottfried Sum Rule violation.

Let us consider now the $x$-dependence of $x\left(\frac{1}{2}(\bar{u}+\bar{d})-\bar{s}\right)$ which has been extracted by Kumano [44] from the experimental data of the E615 Collaboration [60. In Fig. 11 we show the prediction of our model. In analogy to the $x(\bar{d}-\bar{u})$ difference we show separately the effect of the valence quarks in mesons (dashed line). It explains already a significant fraction of the total strange quark suppression. The dotted line shows in addition the effect when the extra perturbative contributions, as described in this section, are included. The slight overestimation of this experimental data may suggest even smaller suppression of the 
perturbative component with respect to that obtained based on the CCFR data.

The net strangeness of the nucleon is zero, which requires: $\int s(x) d x=\int \bar{s}(x) d x$. It has been customarily assumed that $s(x)=\bar{s}(x)$. There is no basic principle that forces one to this assumption other than the fact that it appears as a consequence of a perturbative approach. In this context nonperturbative effects can be of crucial importance. Recently [61], a possible charge $x$-asymmetry of the strange sea has been studied in the chiral GrossNeveu model. Although a large asymmetry has been found, no absolute normalization of the effect was possible. A similar analysis has been performed somewhat earlier by Signal and Thomas [62]. In their approach the result strongly depends on the bag radius. At present, experimental proposals to investigate this effect are under discussion [63]. The presence of (strange meson)-(strange baryon) Fock components in the nucleon wave function suggests the asymmetry in a natural way, as $\bar{s}$ quarks are constituents of 'light' mesons $\left(K, K^{*}\right)$ and $s$ quarks are constituents of 'heavy' baryons $\left(\Lambda, \Sigma, \Sigma^{*}\right)$. Let us estimate the effect in our model. In distinction to Ref. [61], we can estimate the absolute magnitude of the effect as our free parameters have been determined (see the preceding section) by fitting to the experimental data on $p p \rightarrow \Lambda X$ production at high energy. The contributions from the valence strange quarks in baryons and valence anti-strange quarks in mesons are shown in Fig. 12a for two different sets of quark distributions: (a) LO quark distributions in the pion [52] and in the nucleon [64 (dashed line) and (b) NLO quark distributions in the pion [53] and in the nucleon [3] (solid line).

Making the plausible assumption that the effects discussed in the present paper are the only source of the $s-\bar{s}$ asymmetry, the $s-\bar{s}$ difference can be obtained without additional free parameters. Naively one could expect $s>\bar{s}$ in the large- $x$ region and $s<\bar{s}$ in the small $x$ region (see also 61]). We get a rather opposite effect to the naive expectation as seen in Fig. 12b, where we show predictions of our model using the same structure functions as in Fig. 12a. These contributions are rather small in comparison to similar contributions to $\bar{u}$ and $\bar{d}$ quark distributions (see for instance Ref. [7]). As seen from the figure, in comparison to the $\bar{d}-\bar{u}$ difference, $s-\bar{s}$ depends not only on the parton distributions in mesons but 
also on the parton distributions in baryons. This difference can be expressed in terms of the convolution integrals

$$
\begin{aligned}
s(x)-\bar{s}(x)=Z & {\left[\sum_{B} \int_{x}^{1} f_{B M / N}(y) s_{v a l}^{B}\left(\frac{x}{y}\right) \frac{d y}{y}\right.} \\
& \left.-\sum_{M} \int_{x}^{1} f_{M B / N}(y) \bar{s}_{v a l}^{M}\left(\frac{x}{y}\right) \frac{d y}{y}\right] .
\end{aligned}
$$

Eq. (42) shows that the shape of the $s-\bar{s}$ difference depends both on the splitting functions $f$ and valence quark distributions both in $B\left(s_{\text {val }}^{B}\right)$ and $M\left(\bar{s}_{\text {val }}^{M}\right)$. Therefore, the final result depends on two competing effects: (a) $f_{B M / N}(y)$ peaks at $y>0.5$ (for the $\Lambda K$ case, for instance, it is, however, close to $y=1 / 2$ ); (b) the valence quarks in mesons are concentrated at larger $x$ in comparison to the valence quarks in baryons (2 vs. 3 valence quarks, respectively). It is the second effect which wins in our case. Because the $s-\bar{s}$ difference depends on these two competing effects it is somewhat less reliably determined in comparison to the $\bar{d}-\bar{u}$ difference.

The total (anti)strange quark distributions obtained by applying the procedure described above are shown in Fig.12c. In addition we present a decomposition into contributions from the meson's sea and that from the bare nucleon and bare baryons.

Can the strange quark density be obtained in a reliable way more directly from experimental data? It was proposed that the opposite sign dimuon production in the charge current (anti)neutrino DIS can be used as an unique probe of charm particle production and the strange sea content of the nucleon [55]. The mass of the charm quark introduces a threshold suppression into the dimuon production rate which has been overcome in Ref. [55 by applying a simple slow-rescaling model [65]. For comparison in Fig. 12c we show also the result obtained by the CCFR collaboration in the E744 and E770 experiments at Fermilab 555 (dashed line).

By parameterizing the sea quark distributions by the simple $x q_{\text {sea }}(x)=A(1-x)^{\alpha}$ form, the CCFR collaboration has found a quantitative indication that the strange sea in the nucleon is softer than the non-strange sea with $\alpha=9.45$ vs. 6.95 [55] for the non-strange distributions. This observation is consistent with the prediction of our model. In the large- $x$ 
$(>0.25)$ region the non-strange sea is completely dominated by the mesonic (mainly pionic) effects which exhaust practically the total strength obtained from the analysis of DIS data. In contrast in the strange sector the mesonic effects are strongly suppressed by the meson/baryon mass effects. Thus the model predicts less strength in the large- $x$ region in comparison to the non-strange sea quark distributions.

Although we get a rather reasonable agreement with the CCFR dimuon data we do not consider it as an ultimate test of our model. First of all, as already discussed, the mesonic effects are not the only source of the strange quarks at $Q^{2}$ of a few $\mathrm{GeV}^{2}$. Secondly, we want to stress that the interpretation of the CCFR charm production data should be taken with some grain of salt. It has been demonstrated recently [66 that a clear interpretation of the CCFR result in terms of the strange quark distributions is questionable at least in the case

of the ( $W^{ \pm}-$gluon) fusion component of the strange sea and the experimentally measured cross sections may include also contributions of different origin. Furthermore, it is not fully clear whether the slow-rescaling procedure includes the mass effects in a correct way. Our effect of the $s-\bar{s}$ asymmetry is very small. Recently, this asymmetry has been studied by the CCFR collaboration. They have found a very little effect [67], almost consistent with zero, in agreement with our predictions.

\section{THE SPIN OF THE PROTON}

In terms of quark distributions the Ellis-Jaffe Sum Rule can be written as

$$
S_{E J}^{p}=\int_{0}^{1} g_{1}^{p}(x) d x=\frac{1}{2}\left(\frac{4}{9} \Delta u+\frac{1}{9} \Delta d+\frac{1}{9} \Delta s\right)
$$

with $\Delta q(x)$ being the polarized quark distributions

$$
\Delta q=\int_{0}^{1}\left[q^{\uparrow}(x)-q^{\downarrow}(x)+\bar{q}^{\uparrow}(x)-\bar{q}^{\downarrow}(x)\right] d x
$$

where $q^{\uparrow}(x)\left[q^{\downarrow}(x)\right]$ is the quark distribution with flavour $q$ having spin (helicity) parallel (anti parallel) to the nucleon spin. 
It is well known that semileptonic decays of octet baryons can be well described in the Cabibbo model, where one assumes that the axial currents responsible for the semileptonic decays belong to an $S U(3)$ octet. The diagonal matrix elements of these axial currents in this model give the well-known connection to the Ellis-Jaffe Sum Rule. They can be expressed as

$$
\left\langle p, s\left|A_{\mu}^{a}\right| p, s\right\rangle=2 m_{N} s_{\mu} \cdot \Delta q_{a}
$$

with $s_{\mu}$ being the spin-vector $(s \cdot s=-1, p \cdot s=0), A_{\mu}^{a}$ is an axial current defined by $A_{\mu}^{a}=\bar{q} \gamma_{\mu} \gamma^{5} T^{a} q$, with $T^{a}=\lambda^{a} / 2$ being $S U(3)$ generators ( $\lambda^{a}$ are the Gell-Mann matrices), and $T^{0}$ being the identity matrix and

$$
\begin{aligned}
2 \Delta q_{3} & =\Delta u-\Delta d \quad=F+D \\
2 \sqrt{3} \Delta q_{8} & =\Delta u+\Delta d-2 \Delta s=3 F-D . \\
\Delta q_{0} & =\Delta u+\Delta d+\Delta s .
\end{aligned}
$$

The axial coupling constants $F$ and $D$ can be fixed by fitting to semileptonic decay data.

The non-singlet quantities $\Delta q_{3}$ and $\Delta q_{8}$ are multiplied by a known QCD correction factors $\left(1-\alpha_{s} / \pi\right)$ [68,60] (the correction factor for the singlet quantity $\Delta q_{0}$ is different) which for the sake of simplicity we shall suppress throughout this paper.

Taking the EMC result (Eq.2) at face value and assuming $S U(3)$ symmetry(!), in combination with experimental results for the neutron beta decay and the semileptonic decay data for the octet baryons [70], led to an unexpected result

$$
\Delta q_{0}=0.120 \pm 0.094(\text { stat }) \pm 0.138(\text { syst }),
$$

which was consistent with zero. This can be interpreted such that only a small fraction of the proton spin is carried by quarks.

Newer experimental data taken at CERN [15] and SLAC [16] are to some degree controversial. The experimental data for the polarized muon scattering on a deuterium target [15] agrees with the earlier EMC data, with the Ellis-Jaffe integral smaller than the prediction of the Ellis-Jaffe Sum Rule, the fraction of the nucleon spin carried by quarks very small 
and appreciable negative polarization of the strange quarks. On the other hand, the result obtained in polarized electron scattering from the ${ }^{3} \mathrm{He}$ target [16] in the SLAC E142 experiment agrees with the prediction of the Ellis-Jaffe Sum Rule, finding that a relatively large fraction of the nucleon spin is carried by quarks and giving a $\Delta s$ consistent with zero. The experimental results will be summarized later when comparing with results obtained from our model.

In the naive $S U(6)$ quark model (NQM) $F=2 / 3$ and $D=1$, and as a consequence $2 \Delta q_{3}=5 / 3,2 \sqrt{3} \Delta q_{8}=\Delta q_{0}=1$. The 'experimental' result (Eq. 47) is in marked disagreement with the NQM. The NQM neglects the fact that the proton constituents are highly relativistic [71] and interacting [72] objects. Furthermore, not only quarks contribute to the axial-vector singlet but also gluons. Unlike the flavour octet currents, the singlet current $A_{\mu}^{0}$ has an anomalous divergence (for a review see e.g. Ref. [73])

$$
\partial^{\mu} A_{\mu}^{0}=\frac{N_{f} \alpha_{s}}{2 \pi} \operatorname{Tr} \mathcal{G} \tilde{\mathcal{G}}
$$

where $N_{f}$ is the number of active flavours and $\mathcal{G}$ is the gluonic field. The triangle axial anomaly gives rise to an independent gluonic contribution to the flavour singlet axial current [74 82]. As proposed by Efremov-Teryaev [83] and Altarelli-Ross [74] the parton model (PM) values $\Delta u, \Delta d$ and $\Delta s$ should be modified

$$
\left.\Delta q\left(x, Q^{2}\right) \rightarrow \Delta q\left(x, Q^{2}\right)\right|_{P M}-\delta\left(x, Q^{2}\right)
$$

where 84

$$
\delta\left(x, Q^{2}\right)=\frac{\alpha_{s}}{2 \pi} \int_{x}^{1} C^{\Delta g}\left(\frac{x}{y}, Q^{2}\right) \Delta g\left(y, Q^{2}\right) d y
$$

is a correction induced by the axial anomaly. $C^{\Delta g}(z)$ is the probability to find a quark of longitudinal momentum fraction $z$ in a gluon and appropriate polarization. The total gluonic contribution to $\Delta q$ is

$$
\delta \equiv \int_{0}^{1} \delta\left(x, Q^{2}\right)=\frac{\alpha_{s}\left(Q^{2}\right)}{2 \pi} \Delta g\left(Q^{2}\right)
$$


$\Delta g$ in the last formula is the fraction of the proton spin carried by gluons. As a consequence $\Sigma_{P M}$ should be replaced by $\Sigma_{P M}-3 \delta$. The coefficients $C_{\Delta g}$ have been calculated [83, 74, 75,85$]$. On the other hand, $\Delta g$ cannot be calculated and most of the authors tried to adjust $\Delta g$ to describe experimental data (see for instance [86]). There has been also much discussion over the uniqueness of the separation into $\Delta q_{P M}$ and $\delta$ (see for instance [85]). It has been argued [86,87] that the anomaly induces also a non-perturbative gluonic contribution to $\left.\Delta q\left(x, Q^{2}\right)\right|_{P M}$. Although it is very important in understanding the Ellis-Jaffe Sum Rule violation, up to now no definite treatment of the axial anomaly has been worked out. We shall come back to the problem of the anomaly when discussing the mesonic effects.

\section{SEMILEPTONIC DECAYS}

According to our present understanding, the weak semileptonic decays of the octet baryons can be classified into two groups: either a $d$-quark is transformed into a $u$-quark, or an $s$-quark is transformed into a $u$-quark. The matrix elements of the current operators 'responsible' for the semileptonic decays of the baryons belonging to the octet can be parameterized in terms of $q^{2}$-dependent form factors

$$
\begin{aligned}
& \left\langle B_{1}\left|V_{\mu}+A_{\mu}\right| B_{2}\right\rangle=C \bar{u}_{B_{1}}\left[f_{1}\left(q^{2}\right) \gamma_{\mu} \quad+i \frac{f_{2}\left(q^{2}\right)}{m_{1}+m_{2}} \sigma_{\mu \nu} q^{\nu} \quad+\frac{f_{3}\left(q^{2}\right)}{m_{1}+m_{2}} q_{\mu}\right. \\
& \left.+g_{1}\left(q^{2}\right) \gamma_{\mu} \gamma^{5}+i \frac{g_{2}\left(q^{2}\right)}{m_{1}+m_{2}} \sigma_{\mu \nu} q^{\nu} \gamma^{5}+\frac{g_{3}\left(q^{2}\right)}{m_{1}+m_{2}} q_{\mu} \gamma^{5}\right] u_{B_{2}} .
\end{aligned}
$$

The factor $C$ here is the Cabibbo factor $\left(\sin \theta_{C}\right.$ or $\left.\cos \theta_{C}\right)$. At low momentum transfer only two terms, $f_{1}$ (vector) and $g_{1}$ (axial vector), are important. It is customary to extract from experiments the ratio $g_{A} / g_{V}=g_{1}(0) / f_{1}(0)$.

The semileptonic decays can be well described assuming the so-called $S U(3)$ (Cabibbo) model [20]. Within this model the operators for the $d \rightarrow u$ and $s \rightarrow u$ transitions can be expressed in terms of $S U(3)$ group generators

$$
\begin{array}{ll}
d \rightarrow u & A_{\mu}^{1+i 2}=\gamma_{\mu} \gamma^{5}\left(T^{1}+i T^{2}\right), \\
s \rightarrow u & A_{\mu}^{4+i 5}=\gamma_{\mu} \gamma^{5}\left(T^{4}+i T^{5}\right),
\end{array}
$$


which are related to the familiar Gell-Mann matrices $T^{k}=\lambda^{k} / 2$.

Mesonic corrections lead to the renormalization of the axial-vector coupling constants. The vector coupling constants are protected against renormalization by vector current conservation. Mesonic corrections to the axial-vector coupling constants have been taken into account by calculating the loop corrections to the tree level approximation according to Eq.(13). The corresponding diagrams are shown in Fig.2. Preliminary results with the inclusion of intermediate pseudoscalar mesons and associated octet and decuplet baryons have been already presented elsewhere [88].

To perform numerical calculations within our model requires the knowledge of the axial coupling constants for the bare octet and decuplet baryons, vector mesons and transitions octet $\leftrightarrow$ decuplet. The transitions within the baryonic octet are traditionally parameterized by the so-called anti-symmetric $F$ and symmetric $D$ coupling constants. The axial coupling constant for the transition within the decuplet $(H)$ can be fixed by the relation $2\left\langle\Delta^{++}\left|A_{\mu}^{3}\right| \Delta^{++}\right\rangle=H \cdot 2 m_{\Delta} s_{\mu}$. In analogy we define the coupling constant for the interference diagram octet $\longleftrightarrow$ decuplet $(I)$ as $2\left\langle p\left|A_{\mu}^{3}\right| \Delta^{0}\right\rangle=2\left\langle\Delta^{0}\left|A_{\mu}^{3}\right| p\right\rangle=I \cdot 2 \sqrt{m_{N} m_{\Delta}} s_{\mu}$. The matrix elements of axial-vector currents between pseudoscalar mesons vanish. They are, however, finite for vector mesons. Here the structure is analogous to that of the baryonic octet. We denote the corresponding constants as $F V$ and $D V$. Due to parity-conservation the axial coupling constant $F V$ vanishes. A special role is played here by the $\omega$ meson which consists of an octet and a singlet part [30]. For simplicity we neglect couplings to the singlet part, although they are in principle present. The vector meson $\leftrightarrow$ pseudoscalar meson interference terms have an octet structure analogous to the other cases, with coupling constants called $F I$ and $D I$.

In the $S U(6)$ model, i.e. in the model in which all particles are described by their $S U(6)$ wave functions 89], the axial coupling constants can easily be calculated

$$
F=\frac{2}{3}, \quad D=1, \quad H=1, \quad I=4 \frac{\sqrt{2}}{3}, \quad F V=0, \quad D V=1, \quad F I=1, \quad D I=0 .
$$

In Table 3 we present a list of all measured semileptonic decays of the octet baryons. The 
experimental $g_{A} / g_{V}$ ratios are taken from Refs. [70,90. In addition, we present the $S U(3)$ values for $g_{A}$ 's expressed in terms of the symmetric and asymmetric coupling constants and $g_{V}$ 's. Vector current conservation allows one to extract the experimental $g_{A}$ 's, which are presented in the last column. In Table 4 we present the result of our calculations for all possible semileptonic decays. In the column named ' $M C, S U(6)$ ' we show the $g_{A}$ 's calculated within our model with the $S U(6)$ axial-vector coupling constants (Eq. 55) for the bare hadrons. In the column labeled ' $M C, S U(3)^{\prime}$ 'similar results are shown with $F$ and $D$ fitted to the measured values of the axial-vector coupling constants. For comparison, we show the results for the pure (no mesonic corrections) $S U(6)$ model and pure $S U(3)$ model ( $F$ and $D$ fitted to the experimental data from Table 3$)$. The $\chi^{2}$ values presented in the last row for each model give an idea of the fit quality. It is well known that the naive $S U(6)$ model gives a very poor description of the experimental semileptonic decay data. On the other hand, when fitting the $F$ and $D$ parameters an extremely good description of the existing data can be achieved. It is commonly believed that any correction to the $S U(3)$ model may only destroy the nice agreement. Inclusion of mesonic corrections with $S U(6)$ axial coupling constants improves the description of the data dramatically $\left(\chi^{2} / N=4369 \rightarrow \chi^{2} / N=8.5\right)$. An additional variation of the $F$ and $D$ parameters improves the fit further. We cannot allow for variation of the remaining parameters $(H, I, D V, F I)$, because a completely unrestricted fit could result in unphysical values of parameters since the number of experimental data points is limited to only 5 .

In order to demonstrate the effect of different Fock components we present in Table 5 the axial-vector coupling constants $g_{A}$ calculated with the inclusion of intermediate pseudoscalar mesons and octet baryons (oct,ps), with the additional inclusion of decuplet baryons ( $p s)$ and with the additional inclusion of vector mesons (all). In the case of the $S U(6)$ axial coupling constants (Eq. 55) the inclusion of pseudoscalar mesons-octet baryons Fock components improves the quality of the fit to the experimental $g_{A}$ 's in comparison to NQM (see the $\chi^{2}$ values in the last row of the table) tremendously. The additional inclusion of intermediate decuplet baryons deteriorates the fit, increasing the $\chi^{2}$ value. In this context it is worth 
mentioning that the contribution of the octet-decuplet interference terms to the axial-vector

coupling constant for neutron beta-decay $g_{A}^{n \rightarrow p}$ obtained in our calculation is much smaller than in the Cloudy Bag Model 91. Adding vector mesons again improves the fit to the experimental axial-vector coupling constants.

Summarizing, taking into account both pseudoscalar and vector meson corrections does not lead to any significant deviations from the experimental data for semileptonic decays. The quality of the fit is comparable to that of the original Cabibbo model. Therefore, the experimental data for semileptonic decays does not contradict the meson cloud model of the nucleon, which one might naively expect. We do not predict any essential difference between the $S U(3)$ Cabibbo model and our model for any so far unmeasured transitions.

The meson radiative corrections were recently calculated in the framework of baryon chiral perturbation theory including pseudoscalar mesons with both intermediate baryon octet [17] and decuplet [18] components. In order to simplify the calculation the baryon fields were treated there as heavy static fermions. Although the details of the calculation in Refs. [17,18] differ from ours, similar conclusions have been drawn. In comparison to Refs. [17,18, where the experimental error bars where increased in calculating their $\chi^{2}$ values, our agreement with the data is much better. If we increase the error bars as in Refs. [17,,18] we would get $\chi^{2} / N$ of the order of $0.1-0.4$, compared to $\sim 2$ in Refs. [17, 18].

\section{MESON CLOUD EFFECTS ON THE SPIN STRUCTURE OF THE NUCLEON}

In section II we discussed how the matrix elements of axial currents of the dressed nucleon are related to the corresponding matrix elements of the constituents (intermediate baryons and mesons) in the convolution approach. Eq.(46) allows one to relate axial current matrix elements to quark polarizations. Then the Ellis-Jaffe Sum Rule can be expressed in terms of axial-vector matrix elements $\Delta q_{0}, \Delta q_{3}$ and $\Delta q_{8}$ (Eq. 16)

$$
S_{E J}^{p}=\frac{1}{9} \Delta q_{0}+\frac{1}{6} \Delta q_{3}+\frac{1}{6 \sqrt{3}} \Delta q_{8} .
$$


In the same way the Bjorken Sum Rule becomes

$$
S_{B}=\int_{0}^{1}\left[g_{1}^{p}(x)-g_{1}^{n}(x)\right] d x=\frac{1}{3} \Delta q_{3}
$$

which can be expressed in terms of the axial-vector coupling constant for neutron beta decay

$$
S_{B}=\frac{1}{6} g_{A}^{n \rightarrow p}=0.210 .
$$

Practical calculation of axial-vector matrix elements (see Eq. 13) requires additional assumptions about the axial properties of the bare particles. We will assume $\Delta q_{0}=2 \sqrt{3} \Delta q_{8}$ for all non-strange bare particles, which is an extension of the original Ellis-Jaffe Ansatz $\Delta s=0$ [14. In analogy to the previous section we will consider two models: the $S U(6)$ model, with axial coupling constants given by Eq.(55) and the $S U(3)$ model with $F$ and $D$ fitted to the semileptonic data.

In Table 6 we present our results for the Ellis-Jaffe Sum Rule for the proton $S_{E J}^{p}$, the Ellis-Jaffe Sum Rule for the neutron $S_{E J}^{n}$, the Bjorken Sum Rule $S_{B}$ and $\Delta q_{0}$ in both models with different Fock states included: (a) tree level, (b) octet baryons and pseudoscalar mesons (oct, ps), (c) octet and decuplet baryons and pseudoscalar mesons (ps) and (d) the same with an additional inclusion of vector mesons $(a l l)$.

In the case of the $S U(6)$ model inclusion of the (oct,ps) Fock states brings the bare $S U(6)$ value of $5 / 18=0.278$ for the proton Ellis-Jaffe Sum Rule almost half way down to the experimental result [2]. Inclusion of the intermediate decuplet baryons enhances $S_{E J}^{p}$ a little bit, and conversely the addition of vector mesons decreases $S_{E J}^{p}$ slightly. Even with the inclusion of all Fock states we are not able to describe the experimental EMC result for $S_{E J}^{p}$

It would be, however, too naive to expect that our model alone can account for all spin problems; other effects, like the famous axial anomaly (see section V), should be present as well. Currently it is, however, very difficult to give a quantitative estimate of the axial anomaly effect. On the purely phenomenological side one could try to map the $x$ dependence of the axial anomaly contribution to $g_{1}$ by ascribing all the deficiencies of the existing models with respect to the experimental data to the axial anomaly. Then one can write 


$$
g_{1}^{p}=\left.g_{1}^{p}(x)\right|_{\text {model }}+\Delta g_{1}(x), \quad g_{1}^{n}=\left.g_{1}^{n}(x)\right|_{\text {model }}+\Delta g_{1}(x),
$$

where $\Delta g_{1}(x)$ is the contribution of the axial anomaly. In Ref. 92] the unknown contribution was ascribed to the deficiency of the MIT bag model. Such an estimate of the axial anomaly must rely on the model calculation of $\left.g_{1}^{p}(x)\right|_{\text {model }}$ and $\left.g_{1}^{n}(x)\right|_{\text {model }}$. In the present paper we have concentrated on the role of mesonic corrections, therefore considered only very simple models of the bare nucleons (baryons). It has been shown very recently [93] that by combining the mesonic effects calculated according to the present paper together with one version of the Adelaide group bag model [94] an impressive agreement with the EMC [95] and the SLAC [96] data can be obtained at $x>0.1$. The meson cloud scenario leaves therefore much less phenomenological room for the axial anomaly contribution, at least at $x>0.1$. There is still some room left at $x<0.1$, i.e. precisely in the region where the gluonic contribution is expected to play an important role [84].

The Bjorken Sum Rule is independent of the anomaly. Here we get a good agreement with the classical value Eq.(58) (without QCD corrections) when including only intermediate octet baryons with pseudoscalar mesons as well as in the full model. In this context the similarity to the Gottfried Sum Rule is worth noting. In order to compare $S_{B}$ with the experimental results of the SLAC and SMC experiments (see lower panel of Table 6), higher order perturbative corrections have to be included [97]. Perturbative QCD corrections to the Bjorken Sum Rule have been calculated up to $O\left(\left(\alpha_{s} / \pi\right)^{3}\right)$ 98

$$
S_{B}=\frac{1}{6} g_{A}\left[1-\frac{\alpha_{s}\left(Q^{2}\right)}{\pi}-3.5833\left(\frac{\alpha_{s}\left(Q^{2}\right)}{\pi}\right)^{2}-20.2153\left(\frac{\alpha_{s}\left(Q^{2}\right)}{\pi}\right)^{3}\right] .
$$

In the case of the SLAC experiment also higher twist effects probably play an important role [97]. Once higher-order perturbative corrections are taken into account we get a good agreement with the result of the SMC analysis which include all available proton and deuteron data [15].

The classical $S U(6)$ model is attractive due to its simplicity. In practice, in order to describe different data it is necessary to break this symmetry and allow $F$ and $D$ to be fitted to the semileptonic decay data. We also follow this line here. Then we get $S_{E J}^{p}$ closer 
to the experimental EMC result with the 'best' result when only intermediate octet baryons and pseudoscalar mesons are included. In this case $S_{E J}^{n}$ becomes negative, which seems to be the case experimentally [99]. Of course the Bjorken Sum Rule is fulfilled here in all cases, which is due to the relation between $S_{B}$ and neutron beta decay constant $g_{A}^{n \rightarrow p}$ (Eq. 58).

Also the calculated Ellis-Jaffe Sum Rule has to be corrected for perturbative QCD effects. It has been shown 100 that the singlet $\left(\Gamma^{S}\right)$ and the non-singlet $\left(\Gamma^{N S}\right)$ contributions to the integral of the Ellis-Jaffe Sum Rule get different corrections

$$
\Gamma^{S}=\Gamma_{0}^{S}\left(1-\frac{33-8 N_{f}}{33-2 N_{f}} \frac{\alpha_{s}}{\pi}\right), \quad \Gamma^{N S}=\Gamma_{0}^{N S}\left(1-\frac{\alpha_{s}}{\pi}\right)
$$

For example for $Q^{2}=2 \mathrm{GeV}^{2} S_{E J}^{p}$ calculated within our model (see Table 6) is reduced: $0.220 \rightarrow 0.201(S U(6)$, all $)$ and $0.179 \rightarrow 0.163(S U(3)$, all $)$. Here we have taken $\alpha_{s}\left(2 G e V^{2}\right)=0.371$ 101]. Although substantial, the pQCD corrections does not allow to bring our results down to the experimental $S_{E J}^{p}$.

In all models considered, the singlet axial matrix element $\Delta q_{0}$ and $\Delta s$ are far from the one obtained from the EMC analysis(!) [2]. We have stressed the word analysis, as the EMC result is biased by the assumption of the $S U(3)$ symmetry. Since our model violates $S U(3)$ symmetry, $\Delta q_{0}$ does not need to coincide with the value extracted by the EMC. In our model $\Delta q_{0}$ is reduced mainly due to the $\pi N$ Fock state admixture, which causes a partial depolarization of the spin as discussed in Refs. [31]. The depolarization is caused by a cancellation of the spin-preserving and spin-flipping contributions to the $\pi N$ Fock state.

Please note that our model predicts $\Delta s>0$ in contrast to the negative value found from the EMC analysis. In the constituent quark models the nucleon does not contain any internal strangeness and as a consequence $g_{N N \phi}=0$ because $\phi$ is known to be an almost pure $s \bar{s}$ state. This is also the case of the so-called $S U(6)$ related coupling constants 30 which we have used throughout this paper. However, $\phi$ could couple to the nucleon through $K \bar{K}$ very much the same as the $\rho$ meson couples to the nucleon via $\pi \pi$ [102]. Due to its structure the $\phi$ meson could be a good candidate to understand the problem of the "missing" polarized strangeness. To estimate a maximal possible effect of $\phi$ we have taken $g_{N N \phi}$ from 
the vector meson dominance model [103]. Then we get extra $\Delta s \approx 0.005>0$, which is rather small and of opposite sign to the "experimental" $\Delta s$.

\section{CONCLUSIONS}

In the light of recent experiments on the deep-inelastic lepton scattering by nucleons, the understanding of the nucleon structure has become a hot topic in particle and nuclear physics. The recent observation of Gottfried Sum Rule breaking strongly suggests a flavour asymmetry of light sea quarks in the nucleon. The asymmetry occurs in a natural way within the meson cloud model.

The meson cloud model, as in any phenomenological model, requires specification of some external parameters which cannot be calculated at the present time from a more microscopic theory. Analysis of the model over the past few years has shown that the results are rather sensitive to these parameters. At the present stage, it seems to be impossible to derive these parameters from the underlying QCD. In the present paper we have tried to set limits on these parameters by applying the model to high-energy baryon production data. The application of the MCM to these reactions is consistent with its application to the nucleon structure. In practical calculations one has to make an ansatz for the vertex form factors.

It was argued [11] that one should use form factors which guarantee certain symmetries of the longitudinal momentum distribution functions of virtual mesons and baryons. In the present work we followed this approach. In contrast to form factors often used in traditional nuclear physics calculations, the 'symmetric form factors' give a good description of the experimental data. We find an universal cut-off parameter for all Fock states involving octet baryons. This leads to important differences in comparison to other calculations, where universality has been assumed for the $t$-dependent form factors.

After we had fixed the parameters of the vertex form factors in the hadronic sector, we have looked at the consequences in unpolarized and polarized deep-inelastic scattering and for the semileptonic decays. We find a few interesting results. The value of the Gottfried Sum 
Rule obtained from our model $\left(S_{G}=0.224\right)$ is in good agreement with the experimental result obtained by $\mathrm{NMC}\left(S_{G}=0.24 \pm 0.016\right)$. The meson cloud model predicts a $\bar{u}-\bar{d}$ asymmetry concentrated at rather small $x$ in impressive agreement with a recent global fit to the world data on the Drell-Yan and DIS [3].

Since in our model the probability of the strange meson - strange baryon Fock components is substantially reduced in comparison to the non-strange counterparts, we obtain a significant reduction of the strange sea quark distributions. We find $s(x) \neq \bar{s}(x)$ in contrast to the customary assumption $s(x)=\bar{s}(x)$. The effect found is small and differs from naive expectations.

The mesonic corrections lead to renormalization of axial-vector current matrix elements. Large one-loop corrections, which explicitly violate $S U(3)$ symmetry, are in surprisingly good agreement with semileptonic data even with $S U(6)$ axial coupling constants for bare particles.

We find that a significant fraction (20\%) of the nucleon spin is carried by the angular momentum of the mesonic cloud. Although the meson cloud model does not reproduce completely the EMC result for the Ellis-Jaffe Sum Rule, the contribution of the meson cloud cannot be neglected in the total balance of the proton spin, especially in the context of the success of the meson cloud in the explanation of the Gottfried Sum Rule violation and $\bar{u}(x)-\bar{d}(x)$ asymmetry. The presence of higher Fock components seems, however, not sufficient to resolve the spin crisis. Here, probably other effects, to mention only the axial anomaly, play an important role.

Summarizing, in our view the meson cloud model has many attractive features and can account for the description of many experimental data. Here, we have discussed only some aspects. It should be mentioned that this picture of the nucleon provides, in addition, a good description of nucleon electric polarizabilities [104 and that it has a close connection to very successful models of low-energy hadron-hadron scattering [29, 30.

Finally we want to point out that the same model (with the same set of parameters which has been determined from quite different phenomena) turned out to be very successful 13 
in the description of the new NA51 CERN data for the dilepton production in the protonproton and proton-deuteron collisions [5]. The model provides also a good description of the production of slow protons on the "neutron" (extracted from the deuteron target [105]) in the charge-current neutrino and antineutrino DIS reactions [106]. The model discussed here gives also unique predictions for the semi-inclusive DIS $e+p \rightarrow e^{\prime}+n+X$ which will be tested soon at HERA [24. Such a measurement is being prepared by the ZEUS Collaboration which is installing the forward neutron calorimeter [107,25]. A pilot experiment on detecting neutrons from the beam-gas interactions has already been performed and the result is in good agreement with the pion-exchange predictions [25]. Like any model, the meson cloud model requires further testing. In this respect the planned Fermilab experiment measuring the relative dilepton yield in proton-proton and proton-deuteron interactions [6,7] seems to be very important and promising.

\section{Acknowledgments}

Very useful discussion with S.D. Bass, N.N. Nikolaev, A.W. Thomas and V.R. Zoller is gratefully acknowledged. We wish to thank D. von Harrach for drawing our attention to the problem of the $s-\bar{s}$ asymmetry. We are indebted to B. Gibson and Ch. Goodman for carefully reading the manuscript and very useful discussion. This work was supported in part by the Polish KBN grant 224099102.

\section{APPENDIX A: LAGRANGIANS}

Here we present the interaction langragians we employ in our calculations. They are usually used in meson exchange models [29]. $\phi$ denotes a spin-1/2 field $(N), \psi$ a spin-3/2 field $(\Delta)$ of Rarita-Schwinger form; $\pi$ denotes a pseudoscalar field and $\theta$ a vector field $(\rho, \omega)$ :

$$
\begin{aligned}
& \mathcal{L}_{1}=g \cdot i \bar{\phi} \gamma_{5} \pi \phi \\
& \mathcal{L}_{2}=f \cdot \bar{\phi} \partial_{\mu} \pi \psi^{\mu}+h . c ., \\
& \mathcal{L}_{3}=g \cdot \bar{\phi} \gamma_{\mu} \theta^{\mu} \phi+f \cdot \bar{\phi} \sigma_{\mu \nu} \phi\left(\partial^{\mu} \theta^{\nu}-\partial^{\nu} \theta^{\mu}\right),
\end{aligned}
$$




$$
\mathcal{L}_{4}=f \cdot i \bar{\phi} \gamma_{5} \gamma_{\mu} \psi_{\nu}\left(\partial^{\mu} \theta^{\nu}-\partial^{\nu} \theta^{\mu}\right)+h . c .
$$

The anti-symmetric tensor $\sigma_{\mu \nu}$ here is defined as $\sigma_{\mu \nu}=\frac{i}{2}\left[\gamma_{\mu}, \gamma_{\nu}\right]$.

\section{APPENDIX B: VERTEX FUNCTIONS}

Here we collect our results for the helicity dependent vertex functions $V_{I M F}^{\lambda \lambda^{\prime}}\left(y, k_{\perp}^{2}\right)$. $y$ here denotes the longitudinal momentum fraction of the baryon in the nucleon; $\vec{k}_{\perp}=$ $\left(k_{\perp} \cos \varphi, k_{\perp} \sin \varphi\right)$ the transverse momentum of the baryon with respect to the nucleon momentum. The contributions are listed according to particle helicities $\left(1 / 2 \rightarrow \lambda, \lambda^{\prime}\right)$, with $\lambda$ and $\lambda^{\prime}$ being the baryon and meson helicities, respectively.

a.) Transitions for $\mathcal{L}_{1}(N \pi, N \eta, \Sigma K, \Lambda K)$

$$
\begin{array}{lll}
+\frac{1}{2} & 0 & \frac{g}{2} \frac{y m_{N}-m_{B}}{\sqrt{y m_{N} m_{B}}} \\
-\frac{1}{2} & 0 & \frac{g e^{-i \varphi}}{2} \frac{k_{\perp}}{\sqrt{y m_{N} m_{B}}}
\end{array}
$$

b.) Transitions for $\mathcal{L}_{2}\left(\Delta \pi, \Sigma^{*} K\right)$

$$
\begin{array}{lll}
+\frac{3}{2} & 0 & -\frac{f e^{+i \varphi}}{2 \sqrt{2}} \frac{k_{\perp}\left(y m_{N}+m_{B}\right)}{y \sqrt{y m_{N} m_{B}}} \\
+\frac{1}{2} & 0 & \frac{f}{2 \sqrt{6}} \frac{\left(y m_{N}+m_{B}\right)^{2}\left(y m_{N}-m_{B}\right)+k_{\perp}^{2}\left(y m_{N}+2 m_{B}\right)}{y m_{B} \sqrt{y m_{N} m_{B}}} \\
-\frac{1}{2} & 0 & \frac{f e^{-i \varphi}}{2 \sqrt{6}} \frac{k_{\perp}\left[\left(y m_{N}+m_{B}\right)^{2}-3 m_{B}\left(y m_{N}+m_{B}\right)+k_{\perp}^{2}\right]}{y m_{B} \sqrt{y m_{N} m_{B}}} \\
-\frac{3}{2} & 0 & -\frac{f e^{-2 i \varphi}}{2 \sqrt{2}} \frac{k_{\perp}^{2}}{y \sqrt{y m_{N} m_{B}}}
\end{array}
$$

c.) Transitions for $\mathcal{L}_{3}\left(N \rho, N \omega, \Sigma K^{*}, \Lambda K^{*}\right)$ 


$$
\begin{aligned}
& +\frac{1}{2}+1 \quad \frac{g e^{+i \varphi}}{\sqrt{2}} \frac{k_{\perp}}{(1-y) \sqrt{y m_{N} m_{B}}}-f \sqrt{2} e^{+i \varphi} \frac{k_{\perp} m_{N}}{\sqrt{y m_{N} m_{B}}} \\
& +\frac{1}{2} \quad 0 \quad \frac{g}{2} \frac{k_{\perp}^{2}+m_{N} m_{B}(1-y)^{2}-y m_{M}^{2}}{(1-y) m_{M} \sqrt{y m_{N} m_{B}}} \\
& -\frac{f}{2} \frac{\left(y m_{N}-m_{B}\right)\left(y^{2} m_{N}^{2}-y\left(m_{N}^{2}+m_{B}^{2}+m_{M}^{2}\right)+m_{B}^{2}+k_{\perp}^{2}\right)}{y m_{M} \sqrt{y m_{N} m_{B}}} \\
& +\frac{1}{2}-1 \quad \frac{g e^{-i \varphi}}{\sqrt{2}} \frac{y k_{\perp}}{(1-y) \sqrt{y m_{N} m_{B}}}+f \sqrt{2} e^{-i \varphi} \frac{k_{\perp} m_{B}}{\sqrt{y m_{N} m_{B}}} \\
& -\frac{1}{2}+1-\frac{g}{\sqrt{2}} \frac{y m_{N}-m_{B}}{\sqrt{y m_{N} m_{B}}} \\
& -f \sqrt{2} \frac{k_{\perp}^{2}-\left(m_{N}+m_{B}\right)(1-y)\left(y m_{N}-m_{B}\right)}{(1-y) \sqrt{y m_{N} m_{B}}} \\
& -\frac{1}{2} \quad 0 \quad-\frac{g e^{-i \varphi}}{2} \frac{k_{\perp}\left(m_{N}-m_{B}\right)}{m_{M} \sqrt{y m_{N} m_{B}}} \\
& -\frac{f e^{-i \varphi}}{2} \frac{k_{\perp}(1+y)\left(y^{2} m_{N}^{2}-y\left(m_{N}^{2}+m_{B}^{2}+m_{M}^{2}\right)+m_{B}^{2}+k_{\perp}^{2}\right)}{y(1-y) m_{M} \sqrt{y m_{N} m_{B}}} \\
& -\frac{1}{2}-1 \quad f \sqrt{2} e^{-2 i \varphi}-2 \frac{k_{\perp}^{2}}{(1-y) \sqrt{y m_{N} m_{B}}}
\end{aligned}
$$

d.) Transitions for $\mathcal{L}_{4}\left(\Delta \rho, \Sigma^{*} K^{*}\right)$

$$
\begin{array}{lll}
+\frac{3}{2}+1 & -\frac{f e^{+2 i \varphi}}{2} \frac{k_{\perp}^{2}}{y(1-y) \sqrt{y m_{N} m_{B}}} \\
+\frac{3}{2} & 0 & \frac{f e^{+i \varphi}}{\sqrt{2}} \frac{k_{\perp} m_{M}}{(1-y) \sqrt{y m_{N} m_{B}}} \\
+\frac{3}{2}-1 & \frac{f}{2} \frac{m_{N} m_{B}(1-y)^{2}-y m_{M}^{2}}{(1-y) \sqrt{y m_{N} m_{B}}} \\
+\frac{1}{2}+1 & \frac{f e^{+i \varphi}}{2 \sqrt{3}} \frac{k_{\perp}\left[k_{\perp}^{2}-2(1-y) m_{B}^{2}\right]}{y(1-y) m_{B} \sqrt{y m_{N} m_{B}}} \\
+\frac{1}{2} & 0 & -\frac{f}{\sqrt{6}} \frac{m_{M}\left[k_{\perp}^{2}+m_{B}(1-y)\left(y m_{N}-m_{B}\right)\right]}{(1-y) m_{B} \sqrt{y m_{N} m_{B}}} \\
+\frac{1}{2}-1 & \frac{f e^{-i \varphi}}{2 \sqrt{3}} \frac{k_{\perp}\left[y m_{M}^{2}-2 m_{N} m_{B}(1-y)\right]}{(1-y) m_{B} \sqrt{y m_{N} m_{B}}} \\
-\frac{1}{2}+1 & \frac{f}{2 \sqrt{3}} \frac{2(1-y) m_{B} k_{\perp}^{2}+m_{N} m_{M}^{2} y^{3}-(1-y)^{2} m_{B}^{3}}{y(1-y) m_{B} \sqrt{y m_{N} m_{B}}} \\
-\frac{1}{2} & 0 & \frac{f e^{-i \varphi}}{\sqrt{6}} \frac{k_{\perp} m_{M}\left(y m_{N}-(1-y) m_{B}\right)}{(1-y) m_{B} \sqrt{y m_{N} m_{B}}}
\end{array}
$$




$$
\begin{array}{lll}
-\frac{1}{2} & -1 & \frac{f e^{-2 i \varphi}}{2 \sqrt{3}} \frac{k_{\perp}^{2} m_{N}}{(1-y) m_{B} \sqrt{y m_{N} m_{B}}} \\
-\frac{3}{2}+1 & \frac{f e^{-i \varphi}}{2} \frac{k_{\perp} m_{B}(1-y)}{y \sqrt{y m_{N} m_{B}}} \\
-\frac{3}{2} & 0 & 0 \\
-\frac{3}{2} & -1 & 0
\end{array}
$$




\section{REFERENCES}

[1] P. Amaudruz et al. Phys. Rev. Lett. 66 (1991) 2712.

[2] J. Ashman et al. Nucl. Phys. B328 (1989) 1.

[3] A.D. Martin, W.J. Stirling and R.G. Roberts. Phys. Lett. B306 (1993) 145.

[4] A.D. Martin, W.J. Stirling and R.G. Roberts. Phys. Rev. D50 (1994) 6734.

[5] A. Baldit et al. Phys. Lett. B332 (1994) 244.

[6] G.T. Garvey et al. FNAL proposal, P866 (1992) .

[7] A. Szczurek, J. Speth and G.T. Garvey. Nucl. Phys. A570 (1994) 765.

[8] D.A. Ross and C.T. Sachrajda. Nucl. Phys. B149 (1979) 497.

[9] W-Y.P. Hwang and J. Speth. Chin. Jour. Phys. 29 (1991) 461.

[10] A. Szczurek and J. Speth. Nucl. Phys. A555 (1993) 249.

[11] V.R. Zoller. Z. Phys. C53 (1992) 443.

[12] S. Kumano. Phys. Rev. D43 (1991) 3067.

[13] H. Holtmann, N.N. Nikolaev, J. Speth and A. Szczurek. Z. Phys. A (1995) in print.

[14] J. Ellis and R.L. Jaffe. Phys. Rev. D9 (1974) 1444.

[15] D. Adams et al. Phys. Lett. B329 (1994) 399.

[16] P.L. Anthony et al. Phys. Rev. Lett. 71 (1993) 959.

[17] E. Jenkins and A.V. Manohar. Phys. Lett. B255 (1991) 558.

[18] E. Jenkins and A.V. Manohar. Phys. Lett. B259 (1991) 353.

[19] A. Szczurek and H. Holtmann. Acta Phys. Pol. B24 (1993) 1833.

[20] P. Renton. "Electroweak Interaction, An Introduction to the Physics of Quarks and 
Leptons" . Cambridge University Press, Cambridge, 1990.

[21] J.D. Sullivan. Phys. Rev. D5 (1972) 1732.

[22] G.G. Arakelyan, K.G. Boreskov and A.B. Kaǐdalov. Sov. J. Nucl. Phys. 33 (1981) 247.

[23] W-Y.P. Hwang, J. Speth and G.E. Brown. Z. Phys. A339 (1991) 383.

[24] H. Holtmann, G. Levman, N.N. Nikolaev, A. Szczurek and J. Speth. Phys. Lett. B338 (1994) 393.

[25] S. Bhadra et al. 'Test of a forward neutron calorimeter for the ZEUS experiment at HERA', DESY 94-084, (1994) submitted to Nuclear Instruments and Methods.

[26] T.D. Cohen and D.B. Leinweber. Comments Nucl. Part. Phys. 21 (1993) 137.

[27] P.J. Mulders, A.W. Schreiber and H. Meyer. Nucl. Phys. A549 (1992) 498.

[28] V. Dmitrašinović and R. Tegen. Phys. Rev. C46 (1992) 1108.

[29] R. Machleidt, K. Holinde and Ch. Elster. Phys. Rep. 149 (1987) 1.

[30] B. Holzenkamp, K. Holinde and J. Speth. Nucl. Phys. A500 (1989) 485.

[31] V.R. Zoller. Mod. Phys. Lett. A8 (1993) 1113.

[32] W. Melnitchouk and A.W. Thomas. Phys. Rev. D47 (1993) 3794.

[33] G.G. Arakelyan and A.A. Grigoryan. Sov. J. Nucl. Phys. 34 (1981) 745.

[34] S.D. Drell, D.J. Levy and T.M. Yan. Phys. Rev. D1 (1970) 1035.

[35] S. Weinberg. Phys. Rev. 150 (1966) 1313.

[36] S. Schweber. An Introduction to Relativistic Quantum Field Theory . Row-Peterson, New York, 1961.

[37] A.W. Thomas. private discussion . 
[38] W. Melnitchouk, A.W. Schreiber and A.W. Thomas. Phys. Rev. D49 (1994) 1183.

[39] B. Povh and J. Hüfner. Phys. Rev. D46 (1992) 990.

[40] W. Flauger and F. Monning. Nucl. Phys. B109 (1976) 347.

[41] V. Blobel et al. Nucl. Phys. B135 (1978) 379.

[42] R.G.E. Timmermans, Th.A. Rijken and J.J. de Swart. Phys. Lett. B257 (1991) 227.

[43] R.G.E. Timmermans, Th.A. Rijken and J.J. de Swart. Phys. Rev. D45 (1992) 2288.

[44] S. Kumano. Phys. Rev. D43 (1991) 59.

[45] E.G. Boos et al. Nucl. Phys. B151 (1979) 193.

[46] D. Brick et al. Phys. Rev. D21 (1980) 632.

[47] E.M. Henley and G.A. Miller. Phys. Lett. B251 (1990) 453.

[48] A. Signal, A.W. Schreiber and A.W. Thomas. Mod. Phys. Lett. A6 (1991) 271.

[49] Bo-Qiang Ma, A. Schäfer, W. Greiner. Phys. Rev. D47 (1993) 51.

[50] W. Melnitchouk and A.W. Thomas. Phys. Rev. D47 (1993) 3783.

[51] C. Foudas et al. Phys. Rev. Lett. 64 (1990) 1207.

[52] J. Badier et al. Z. Phys. C18 (1983) 281.

[53] P.J. Sutton, A.D. Martin, R.G. Roberts and W.J. Stirling. Phys. Rev. D45 (1992) 2349 .

[54] S.R. Mishra et al. Phys. Rev. Lett. 24 (1992) 3499.

[55] S.A. Rabinowitz et al CCFR. Phys. Rev. Lett. 70 (1993) 134.

[56] G. Altarelli and G. Parisi. Nucl. Phys. B126 (1977) 298.

[57] M. Glück, E. Reya and A. Vogt. Z. Phys. C53 (1992) 651. 
[58] I. Abt et al. Nucl. Phys. B407 (1993) 515.

[59] M. Derrick et al. Phys. Lett. B316 (1993) 412.

[60] J.G. Heinrich et al. Phys. Rev. Lett. 63 (1989) 356.

[61] M. Burckardt, B.J. Warr. Phys. Rev. D45 (1992) 958.

[62] A.I. Signal and A.W. Thomas. Phys. Lett. B191 (1987) 205.

[63] D. von Harrach. Invited talk at the Mainz Workshop on the European Electron Facility, Mainz, 16-19 October 1992, to be published in the Proceedings. .

[64] J.F. Owens. Phys. Lett. B266 (1991) 126.

[65] H. Georgi and H.D. Politzer. Phys. Rev. D14 (1976) 1829.

[66] V. Barone, M. Genovese, N.N. Nikolaev, E. Predazzi and B.G. Zakharov. Phys. Lett. B321 (1994) 137.

[67] A.O. Bazarko et al. preprint Nevis R\#1502 (1994) .

[68] J. Kodaira, S. Matsuda, T. Muta, T. Uematsu and K. Sasaki. Phys. Rev. D20 (1979) 627.

[69] J. Kodaira, S. Matsuda, K.Sasaki and T. Uematsu. Nucl. Phys. B159 (1979) 99.

[70] Particle Data Group. Phys. Rev. D45 (1992) 1.

[71] A. Chodos, R.L. Jaffe, K. Johnson and C.B. Thorn. Phys. Rev. D10 (1974) 2599.

[72] M. Takizawa and W. Weise. Phys. Lett. B268 (1991) 323.

[73] A. Shifman. Phys. Rep. 209 (1991) 341.

[74] G. Altarelli and G.G. Ross. Phys. Lett. B212 (1988) 391.

[75] R.D. Carlitz and J.C. Collins and A.H. Mueller. Phys. Lett. B214 (1988) 229. 
[76] R.L. Jaffe and A. Manohar. Nucl. Phys. B337 (1990) 509.

[77] R. Johnson, N.W. Park, J. Schechter, V.Soni and H. Weigel. Phys. Rev. D42 (1990) 2998.

[78] A.V. Efremov, J. Soffer and O.V. Teryaev. Nucl. Phys. B246 (1990) 87.

[79] A. Schäfer. Nucl. Phys. A527 (1991) 559c.

[80] H. Fritzsch. Phys. Lett. B256 (1991) 75.

[81] M. Glück and E. Reya. Phys. Lett. B270 (1991) 65.

[82] A.V. Efremov and J. Soffer and N.A. Törnqvist. Phys. Rev. D44 (1991) 1369.

[83] A.V. Efremov and O.V. Teryaev. Dubna preprint E2-88-287 (1988) .

[84] S.D. Bass and A.W. Thomas. J. Phys. G19 (1993) 925.

[85] S.D. Bass, B.L. Ioffe, N.N. Nikolaev and A.W. Thomas. J. Moscow Phys. Soc. 1 (1991) 317.

[86] T. Gehrmann, W.J. Stirling. Z. Phys. C65 (1995) 461.

[87] S.D. Bass. Phys. Lett. B342 (1995) 233.

[88] A. Szczurek. invited talk at the workshop, 'Exclusive Reactions at High Momentum Transfer', 23-26 June 1993 Elba, World Scientific, edited by C.Carlson, P.Stoler, M.Taiuti, Singapore 1994, p. 92. .

[89] F. Close. An Introduction to Quarks and Partons . Academic Press, London, 1979.

[90] P. Franzini et al. Phys. Rev. D6 (1972) 2417.

[91] A.W. Schreiber and A.W. Thomas. Phys. Lett. B215 (1988) 141.

[92] S.D. Bass and A.W. Thomas. Phys. Lett. B312 (1993) 345.

[93] F.M. Steffens, H. Holtmann and A.W. Thomas. Phys. Lett. B358 (1995) 139. 
[94] F.M. Steffens and A.W. Thomas. Nucl. Phys. A568 (1994) 798.

[95] J. Ashman et al. Nucl. Phys. B328 (1989) 1.

[96] K. Abe et al. preprint SLAC-PUB-6508 (1994) .

[97] J. Ellis and M. Karliner. Phys. Lett. B341 (1995) 397.

[98] S.A. Larin and J.A.M. Vermaseren. Phys. Lett. B259 (1991) 345.

[99] B. Adeva et al SMC. Phys. Lett. B302 (1993) 533.

[100] J. Kodaira. Nucl. Phys. B165 (1980) 129.

[101] J. Ellis and M. Karliner. Phys. Lett. B313 (1993) 131.

[102] G. Höhler and E. Pietarinen. Nucl. Phys. B95 (1975) 210.

[103] B.L. Ioffe, V.A. Khoze and L.N. Lipatov. Hard Processes . North Holland, Amsterdam, 1984.

[104] V. Bernard, N. Kaiser and U.G. Meissner. Nucl. Phys. B373 (1992) 346.

[105] G.D. Bosveld, A.E.L. Dieperink and A.G. Tenner. Phys. Rev. C49 (1994) 2379.

[106] A. Szczurek, G.D. Bosveld and A.E.L. Dieperink. Groningen preprint KVI-1096 (1994)

[107] G. Levman und F. Furutani. ZEUS-Note 92-107 (1992) . 


\section{FIGURES}

FIG. 1. Deep inelastic scattering on a virtual meson (a) or baryon (b).

FIG. 2. Corrections to an axial current in the MCM.

FIG. 3. One-Boson-Exchange diagrams for $n$ and $\Lambda$ production.

FIG. 4. Differential cross section for $p p \rightarrow n X$ (Fig.4a) and $p p \rightarrow \Lambda X$ production 40,41(Fig.4b). Shown are the OBE contributions: pseudoscalar mesons (dashed), vector mesons (dotted) and their sum (solid).

FIG. 5. The same as in Fig.4a for an traditional dipole form factor with $\Lambda=1.2 \mathrm{GeV}$.

FIG. 6. One-Boson-Exchange diagram for $\Delta^{++}$production.

FIG. 7. Differential cross section for $p p \rightarrow \Delta^{++} X$ production. The experimental spectra shown are from Ref. [45]. The dashed line corresponds to $\pi$-exchange, the dotted line to $\rho$-exchange and the solid line to the sum of both contributions. The two different scales correspond to different background subtractions. The calculated results are shown with respect to the right scale.

FIG. 8. The $y$-integrated $p p \rightarrow \Delta^{++}$and $\bar{p} p \rightarrow \Delta^{++}$cross sections 45, 46]: $\pi$-exchange (dashed), $\rho$-exchange (dotted) and their sum (solid).

FIG. 9. The result of the fit of the $R_{\text {sea }}$ factor to the CCFR experimental data for $\bar{q}(x)$. 
FIG. 10. (a) The $x(\bar{d}-\bar{u})$ asymmetry as a function of the Bjorken variable $x$ calculated within our model with three different pion structure functions: NA3 LO [52] (dotted), NLO [53] (solid) and of the GRV model [57] (dashed).

(b) The same for recent experimental parameterizations with explicit inclusion of the flavour asymmetry: $\operatorname{MSR}\left(D_{0}^{\prime}\right)[3]$ (dotted), $\operatorname{MSR}\left(D_{-}^{\prime}\right)$ [3] (dash-dotted) and the newest parameterization of the same group MSR(A) [ [ (dashed). The full line (MCM) is again a prediction of our model.

FIG. 11. The difference $x\left(\frac{1}{2}(\bar{u}+\bar{d})-\bar{s}\right)$ as function of $x$. The points with error bars have been extracted by Kumano using results from the E615 collaboration 44. The dashed line is the MCM result obtained with valence quarks only. Inclusion of the sea-quarks in mesons and baryons (as described in the text) gives the solid line.

FIG. 12. The contributions of $\bar{s}_{v a l}^{M}$ and $s_{v a l}^{B}$ to the nucleon $\bar{s}(x)$ and $s(x)$ quark distributions, respectively, for LO parameterizations [52], [64] (dashed) and NLO parameterizations [53], [3] (solid).

(b) $s(x)-\bar{s}(x)$ for two sets of structure functions: $\pi$ [52] $+\mathrm{N}$ [64 (dashed) and $\pi$ [53] $+\mathrm{N}$ MSR $S_{0}^{\prime}[3]$ (solid).

(c) The contributions from $s_{\text {sea }}^{M}, s_{\text {sea }}^{B}$ and $s_{\text {sea }}^{N^{\prime}}$ (bare nucleon) to the strange sea of the nucleon. Details are described in the text. The structure functions taken from Refs. [3], [53]. For comparison we show the "experimental" result of the CCFR collaboration (dashed line). 


\section{TABLES}

\begin{tabular}{lccc}
\hline \hline & exponential & monopole & dipole \\
\hline$\Lambda_{\pi}(\mathrm{GeV})$ & 1.08 & 1.9 & 2.2 \\
$\Lambda_{\rho}(\mathrm{GeV})$ & 1.08 & 1.4 & 2.0 \\
\hline$f_{p \pi^{0} / p}$ & 0.204 & 0.210 & 0.219 \\
$\Delta f_{p \pi^{0} / p}$ & 0.017 & 0.015 & 0.014 \\
$f_{p \rho^{0} / p}$ & 0.062 & 0.075 & 0.060 \\
$\Delta f_{p \rho^{0} / p}$ & -0.038 & -0.042 & -0.034 \\
\hline \hline
\end{tabular}

TABLE I. Cut-off parameters for the different functional forms of form factors obtained by fitting to the neutron production data and the corresponding first moments of splitting functions.

\begin{tabular}{llcc}
\hline \hline model & $Z$ & $A_{\bar{u}-\bar{d}}$ & $S_{G}$ \\
\hline tree & 1 & 0 & 0.333 \\
oct, ps & 0.755 & -0.155 & 0.230 \\
ps & 0.697 & -0.119 & 0.254 \\
all & 0.580 & -0.163 & 0.224 \\
\hline \hline
\end{tabular}

TABLE II. The Gottfried Sum Rule and the $\bar{u}-\bar{d}$ asymmetry in different models. Model $(o c t, p s)$ includes all Fock states with octet baryons and pseudoscalar mesons, ( $p s)$ all Fock states with octet and decuplet baryons and pseudoscalar mesons and (all) all discussed Fock states. The experimental value of the Gottfried Sum Rule is $0.24 \pm 0.016$ []. 


\begin{tabular}{lcccccc}
\hline \hline & decay & & $g_{A} S U(3)$ & $g_{V} S U(3)$ & $g_{A} / g_{V} \exp$ & $g_{A} \exp$ \\
\hline$n$ & $\rightarrow$ & $p$ & $F+D$ & 1 & $1.2573 \pm 0.0028$ & $1.273 \pm 0.0028$ \\
$\Sigma^{-}$ & $\rightarrow$ & $\Lambda$ & $2 D / \sqrt{6}$ & 0 & & $0.60 \pm 0.03$ \\
$\Lambda$ & $\rightarrow$ & $p$ & $-(3 F+D) / \sqrt{6}$ & $-\sqrt{3 / 2}$ & $0.718 \pm 0.015$ & $-0.857 \pm 0.018$ \\
$\Xi^{-}$ & $\rightarrow$ & $\Lambda$ & $(3 F+D) / \sqrt{6}$ & $\sqrt{3 / 2}$ & $0.25 \pm 0.05$ & $0.31 \pm 0.06$ \\
$\Sigma^{-}$ & $\rightarrow$ & $n$ & $D-F$ & -1 & $-0.34 \pm 0.05$ & $0.34 \pm 0.05$ \\
\hline \hline
\end{tabular}

TABLE III. List of all measured semileptonic decays of baryons and the corresponding coupling constants in the $S U(3)$ model. The data were taken from [70] and [90] (for $\Sigma^{-} \rightarrow \Lambda$ ). 


\begin{tabular}{lccccccc}
\hline \hline & decay & & $S U(6)$ & $S U(3)$ & $M C, S U(6)$ & $M C, S U(3)$ & $g_{A}$ exp \\
\hline$n$ & $\rightarrow$ & $p$ & 1.67 & 1.257 & 1.241 & 1.257 & $1.2573 \pm 0.0028$ \\
$\Sigma^{+}$ & $\rightarrow$ & $\Lambda$ & 0.82 & 0.67 & 0.66 & 0.74 & \\
$\Sigma^{-}$ & $\rightarrow$ & $\Sigma^{0}$ & 0.94 & 0.62 & 0.77 & 0.64 & \\
$\Sigma^{-}$ & $\rightarrow$ & $\Lambda$ & 0.82 & 0.67 & 0.65 & 0.75 & $0.60 \pm 0.03$ \\
$\Sigma^{+}$ & $\rightarrow$ & $\Sigma^{0}$ & -0.94 & -0.62 & -0.77 & -0.64 & \\
$\Xi^{-}$ & $\rightarrow$ & $\Xi^{0}$ & 0.33 & 0.38 & 0.27 & 0.49 & \\
\hline$\Lambda$ & $\rightarrow$ & $p$ & -1.22 & -0.87 & -0.96 & -0.89 & $-0.857 \pm 0.018$ \\
$\Sigma^{0}$ & $\rightarrow$ & $p$ & 0.24 & 0.27 & 0.19 & 0.31 & \\
$\Sigma^{-}$ & $\rightarrow$ & $n$ & 0.33 & 0.38 & 0.27 & 0.49 & $0.34 \pm 0.05$ \\
$\Xi^{0}$ & $\rightarrow$ & $\Sigma^{+}$ & 1.67 & 1.26 & 1.37 & 1.39 & \\
$\Xi^{-}$ & $\rightarrow$ & $\Lambda$ & 0.41 & 0.20 & 0.35 & 0.16 & $0.31 \pm 0.06$ \\
$\Xi^{-}$ & $\rightarrow$ & $\Sigma^{0}$ & 1.18 & 0.89 & 0.97 & 0.98 & \\
\hline & $\chi^{2} / N$ & & 4369 & 2.0 & 8.5 & 6.5 & \\
\hline \hline
\end{tabular}

TABLE IV. A list of all possible semileptonic decays of baryons within the nucleon octet. The axial couplings $g_{A}$ has been calculated at the tree-level in the $S U(6)$ model $(F=2 / 3$ and $D=1)$ and in the $S U(3)$ model with $F$ and $D(F=0.44, D=0.82)$ fitted. Also shown are the results with explicit inclusion of the mesonic corrections with $S U(6)$ and $S U(3)$ axial-vector constants for the bare baryons. In the $S U(3)$ case we find $F=0.53$ and $D=1.15$. 


\begin{tabular}{|c|c|c|c|c|c|c|c|c|}
\hline \multirow{2}{*}{\multicolumn{2}{|c|}{ decay }} & & \multicolumn{3}{|c|}{$S U(6)$} & \multicolumn{3}{|c|}{$S U(3)$} \\
\hline & & & oct,ps & $p s$ & all & oct,ps & $p s$ & all \\
\hline$n$ & $\rightarrow$ & $p$ & 1.247 & 1.454 & 1.241 & 1.257 & 1.257 & 1.257 \\
\hline$\Sigma^{-}$ & $\rightarrow$ & $\Lambda$ & 0.70 & 0.77 & 0.65 & 0.80 & 0.71 & 0.75 \\
\hline$\Lambda$ & $\rightarrow$ & $p$ & -0.98 & -1.08 & -0.96 & -0.89 & -0.87 & -0.89 \\
\hline$\Xi^{-}$ & $\rightarrow$ & $\Lambda$ & 0.37 & 0.38 & 0.35 & 0.17 & 0.40 & 0.16 \\
\hline \multirow[t]{2}{*}{$\Sigma^{-}$} & $\rightarrow$ & $n$ & 0.27 & 0.32 & 0.27 & 0.50 & 0.40 & 0.49 \\
\hline & $\chi^{2} / N$ & & 14.7 & 1026 & 8.5 & 13.2 & 3.5 & 6.5 \\
\hline
\end{tabular}

TABLE V. The axial-vector coupling constants for the measured semileptonic decays in different models: octet baryons with pseudoscalar mesons (oct,ps), octet and decuplet baryons with pseudoscalar mesons $(p s)$ and with vector mesons in addition $(a l l)$. The axial coupling constants for the $S U(3)$ case are: $F=0.53, D=1.15$ for (oct,ps) and $F=0.48, D=0.91$ for $p s$ and $F=0.53, D=1.15$ for all. 


\begin{tabular}{|c|c|c|c|c|c|}
\hline & $S_{E J}^{p}$ & $S_{E J}^{n}$ & $S_{B}$ & $\Delta q_{0}$ & $\Delta s$ \\
\hline$S U(6)$ tree & 0.278 & 0. & 0.278 & 1. & 0. \\
\hline oct,ps & 0.212 & 0.004 & 0.208 & 0.779 & 0.004 \\
\hline$p s$ & 0.233 & -0.010 & 0.243 & 0.804 & 0.002 \\
\hline all & $0.220^{*)}$ & 0.011 & $0.209^{* *)}$ & 0.846 & 0.017 \\
\hline$S U(3)$ tree & 0.173 & -0.037 & 0.210 & 0.489 & 0. \\
\hline oct, ps & 0.154 & -0.056 & 0.210 & 0.356 & 0.003 \\
\hline$p s$ & 0.169 & -0.041 & 0.210 & 0.461 & 0.001 \\
\hline all & $0.179^{*)}$ & -0.031 & $0.210^{* *)}$ & 0.541 & 0.018 \\
\hline$E M C$ [2] & 0.126 & - & - & 0.120 & -0.19 \\
\hline$\left[Q^{2}=10.7 \mathrm{GeV}^{2}\right]$ & $\pm 0.01 \pm 0.015$ & & & $\pm 0.094 \pm 0.138$ & $\pm 0.032 \pm 0.046$ \\
\hline E142 [16] & - & -0.022 & 0.146 & 0.57 & -0.01 \\
\hline$\left[Q^{2}=2 G e V^{2}\right]$ & & \pm 0.011 & $\pm 0.021(+\mathrm{EMC})$ & \pm 0.11 & \pm 0.06 \\
\hline$S M C$ [15] & 0.136 & - & - & 0.22 & -0.12 \\
\hline$\left[Q^{2}=5 G e V^{2}\right]$ & $\pm 0.011 \pm 0.011$ & & & $\pm 0.10 \pm 0.10$ & $\pm 0.04 \pm 0.04$ \\
\hline all [15] & 0.142 & -0.069 & 0.204 & 0.27 & -0.10 \\
\hline$\left[Q^{2}=5 G e V^{2}\right]$ & $\pm 0.008 \pm 0.011$ & \pm 0.025 & \pm 0.029 & $\pm 0.08 \pm 0.10$ & $\pm 0.03 \pm 0.04$ \\
\hline
\end{tabular}

TABLE VI. The Ellis-Jaffe Sum Rule for proton and neutron, the Bjorken Sum Rule and the axial flavour singlet constant obtained with inclusion of different Fock states (see text). In the lowest panel we have collected recent experimental data of EMC, E142 and SMC collaborations. The theoretical values of $S_{E J}$ and $S_{B}$ are not corrected for pQCD (!).

*) For $Q^{2}=5 \mathrm{GeV}^{2}, S_{E J}^{p}$ corrected for pQCD effects according to 100 is $0.21(S U(6))$ and 0.17 $(S U(3))$.

**) The pQCD corrections up to $\alpha_{s}^{3}$ give correspondingly $S_{B}=0.19$ in both $S U(6)$ and $S U(3)$ model. 

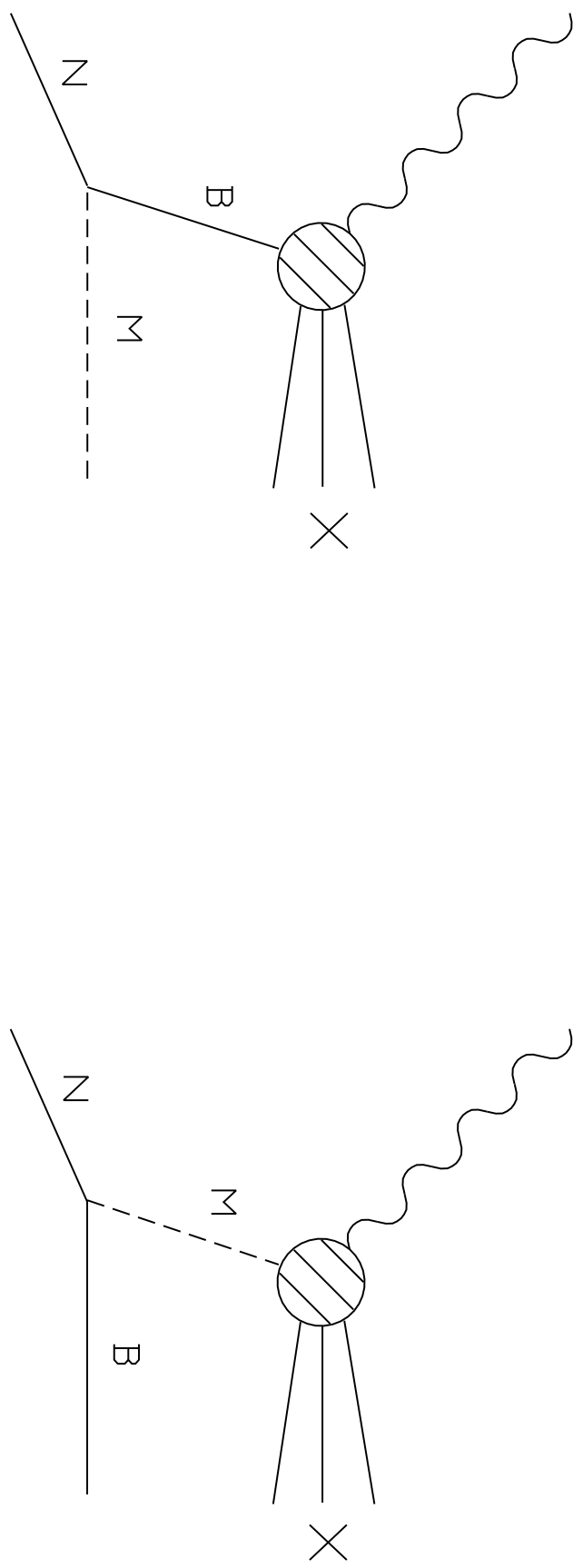


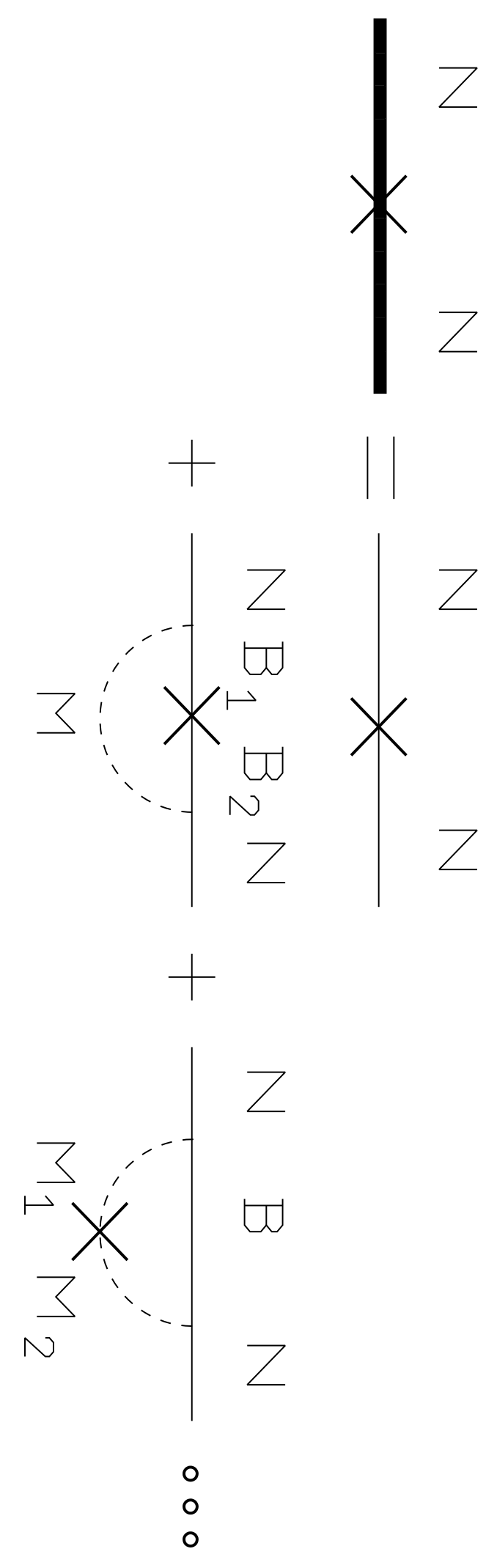



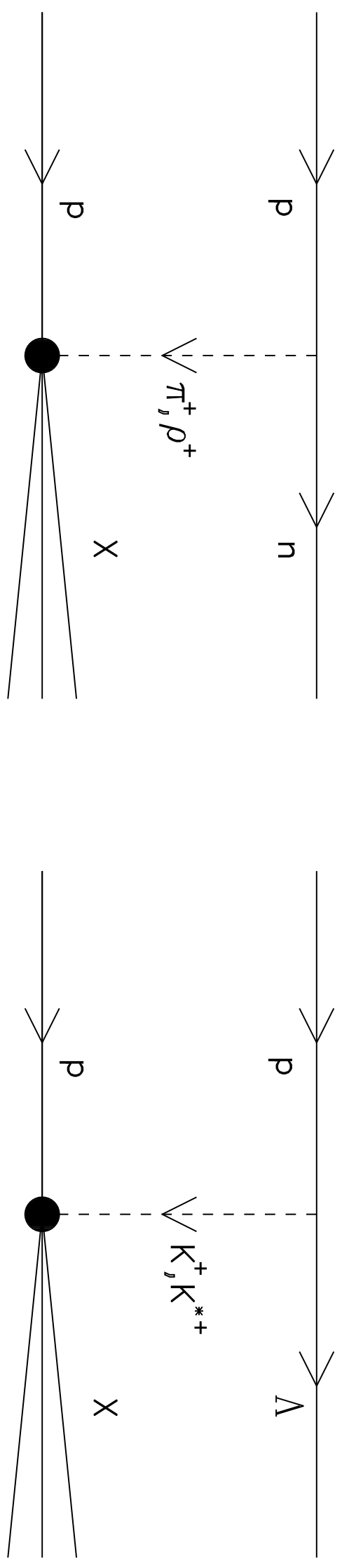

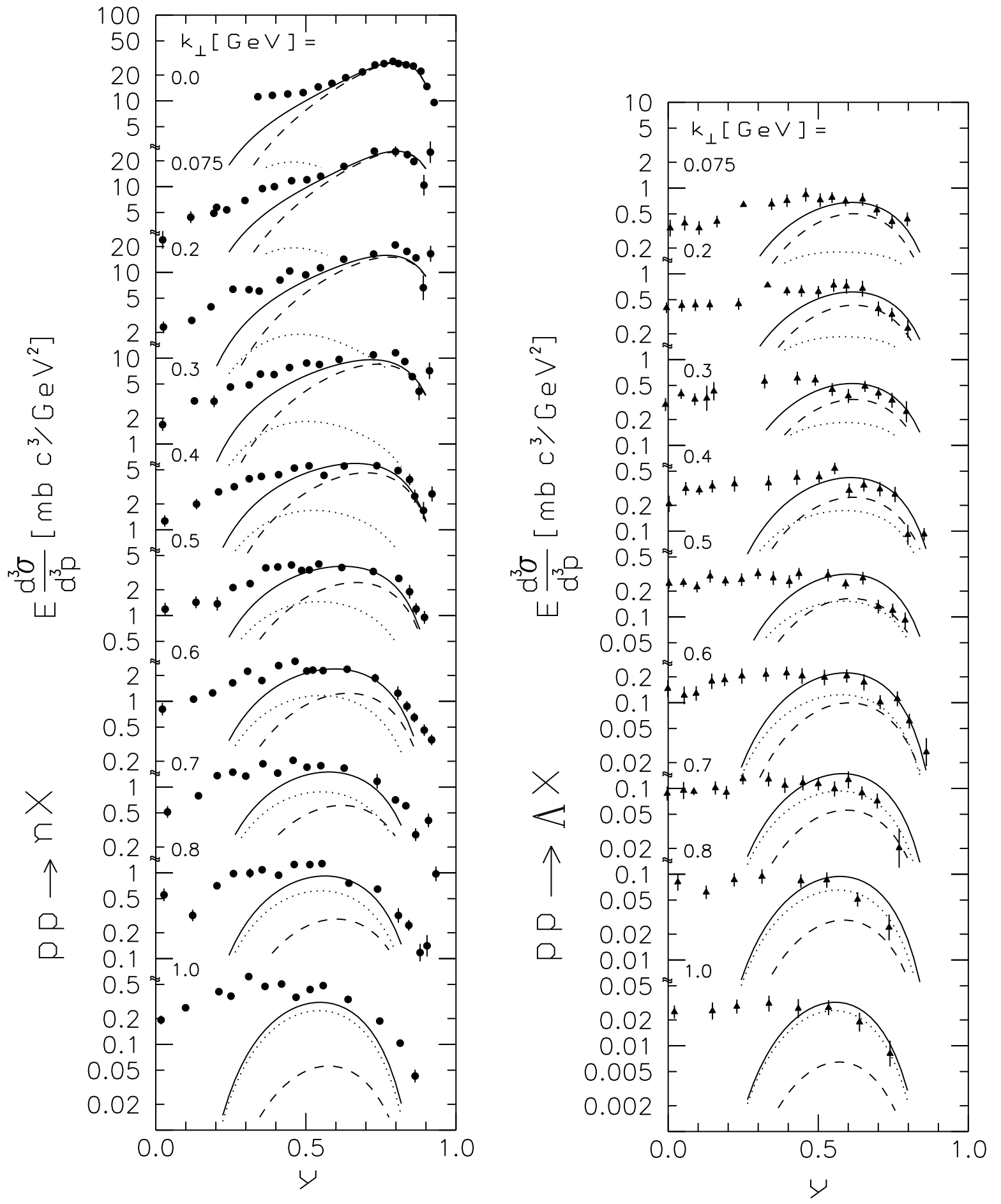


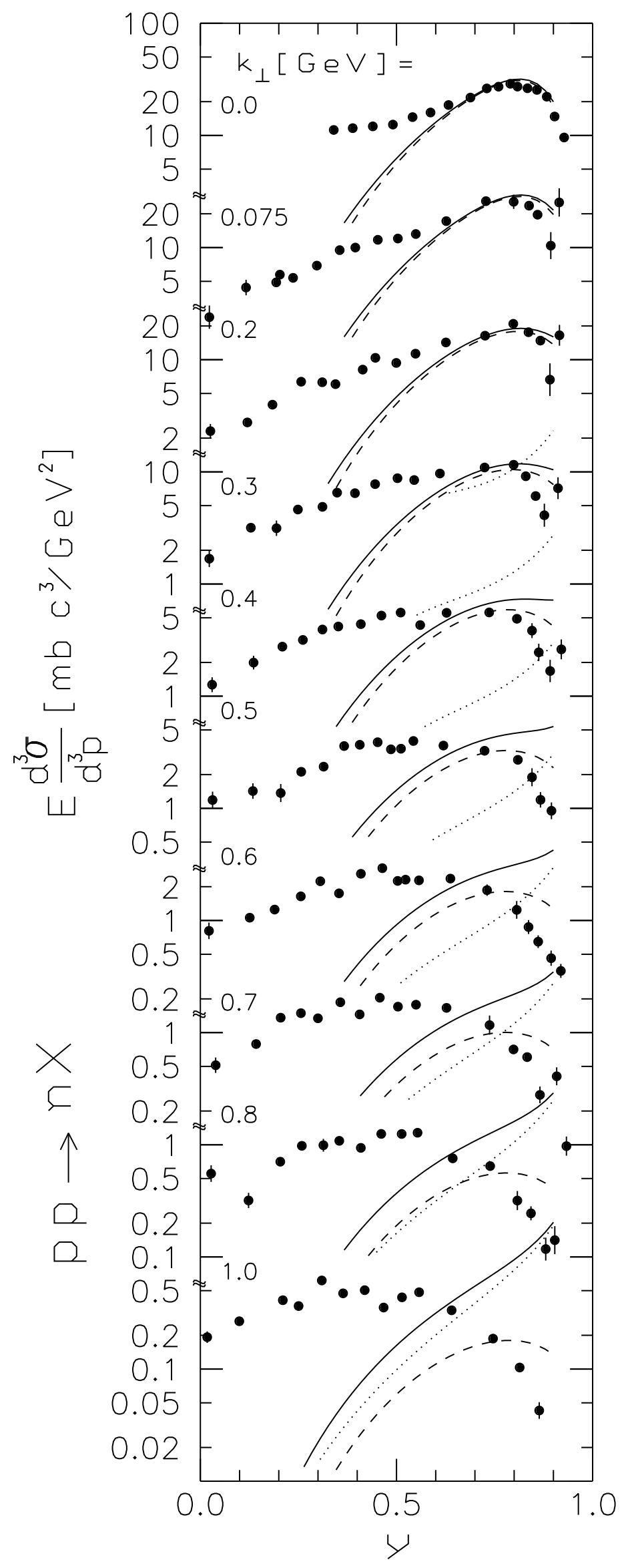




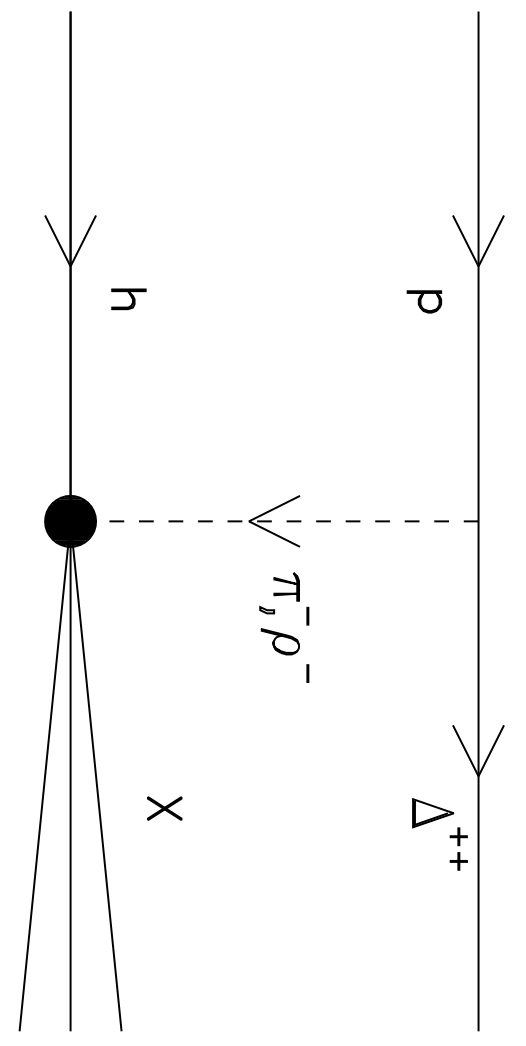




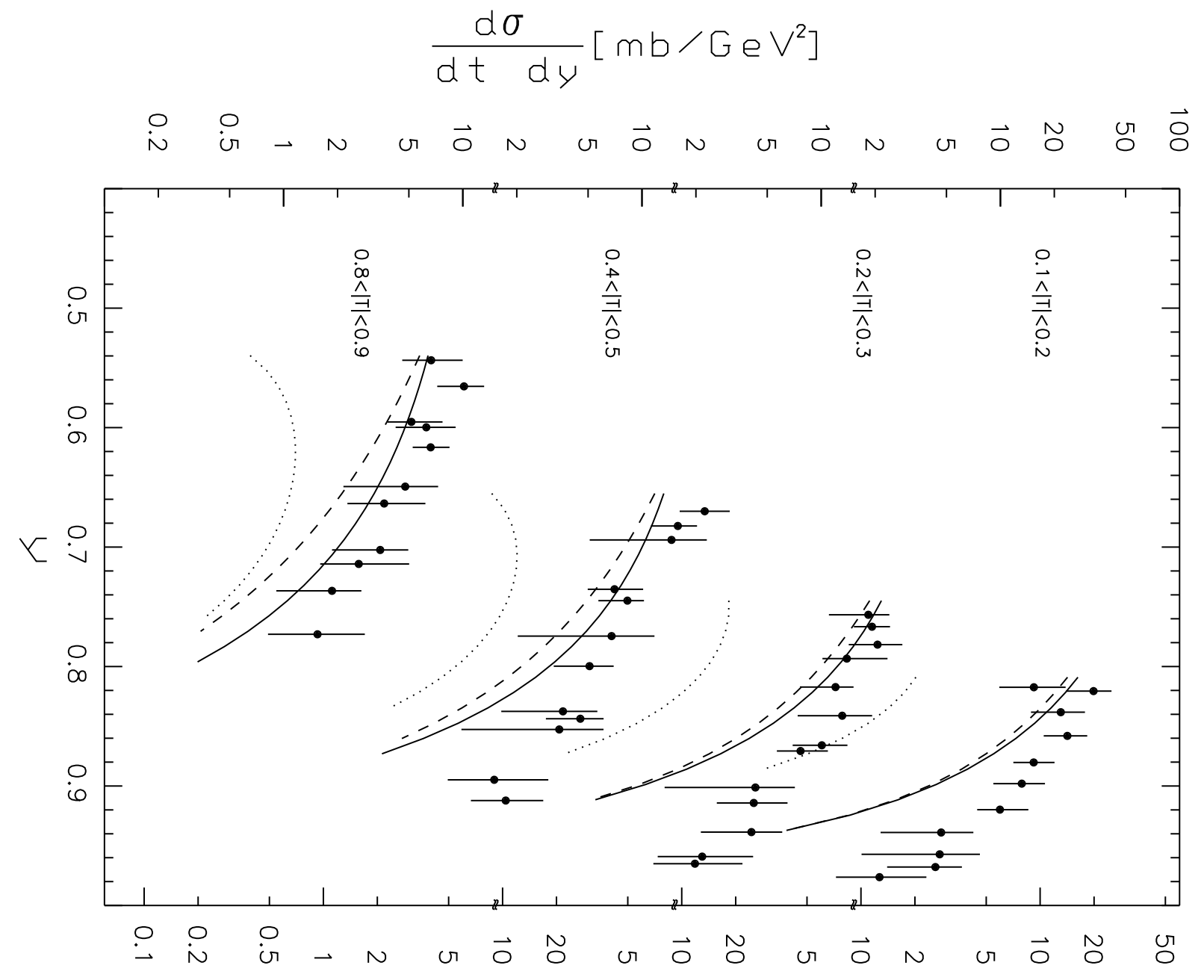



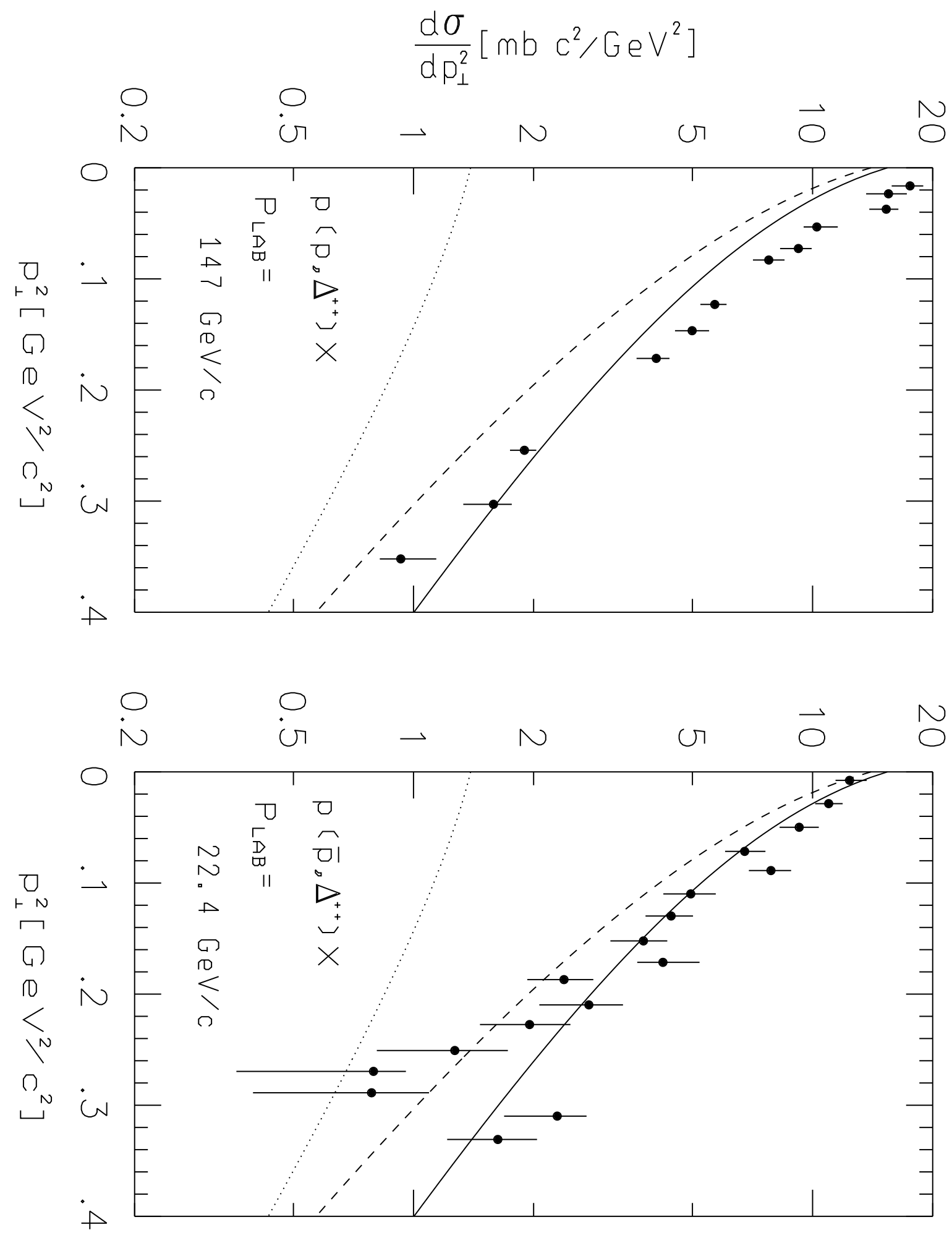


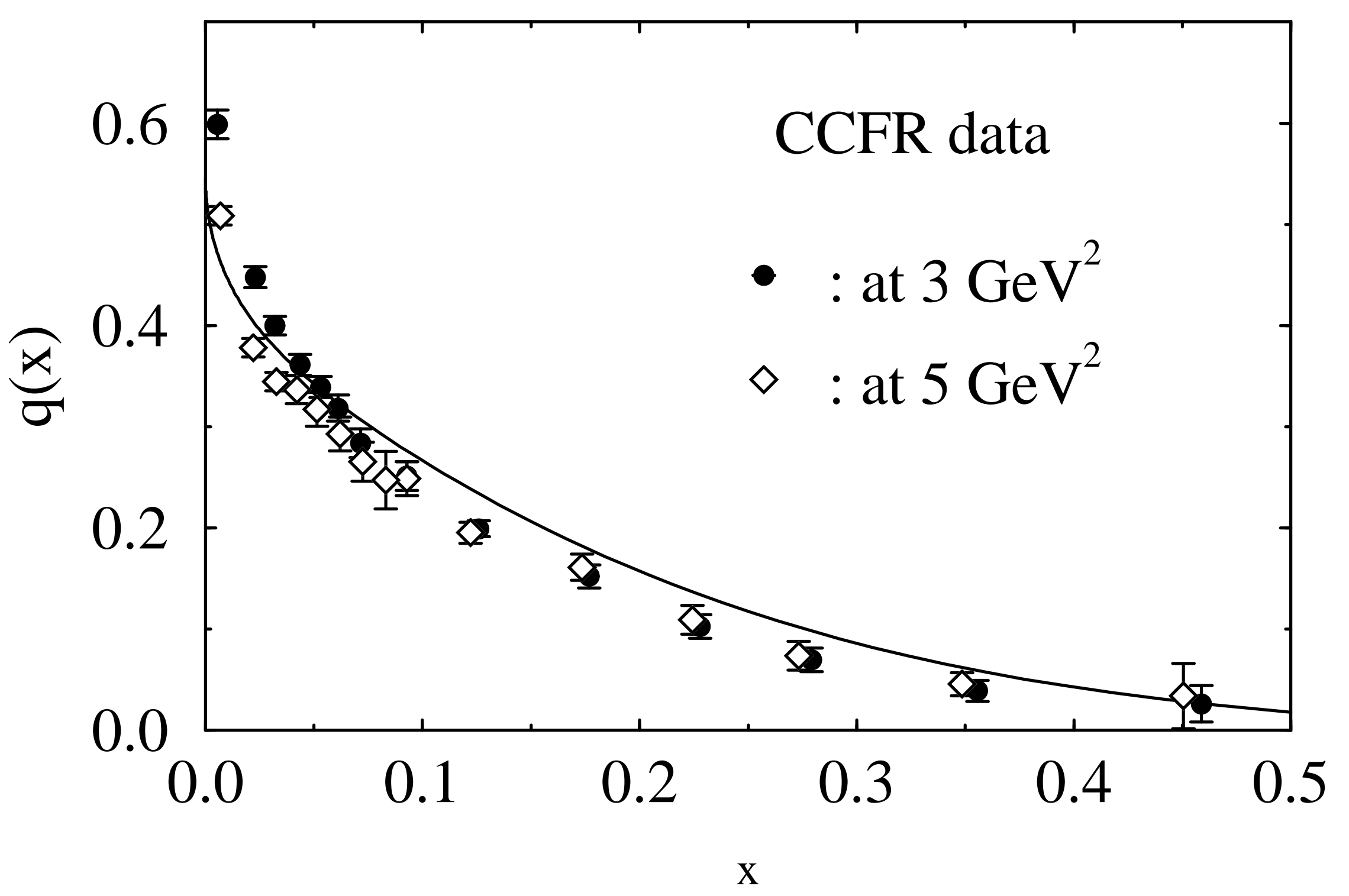




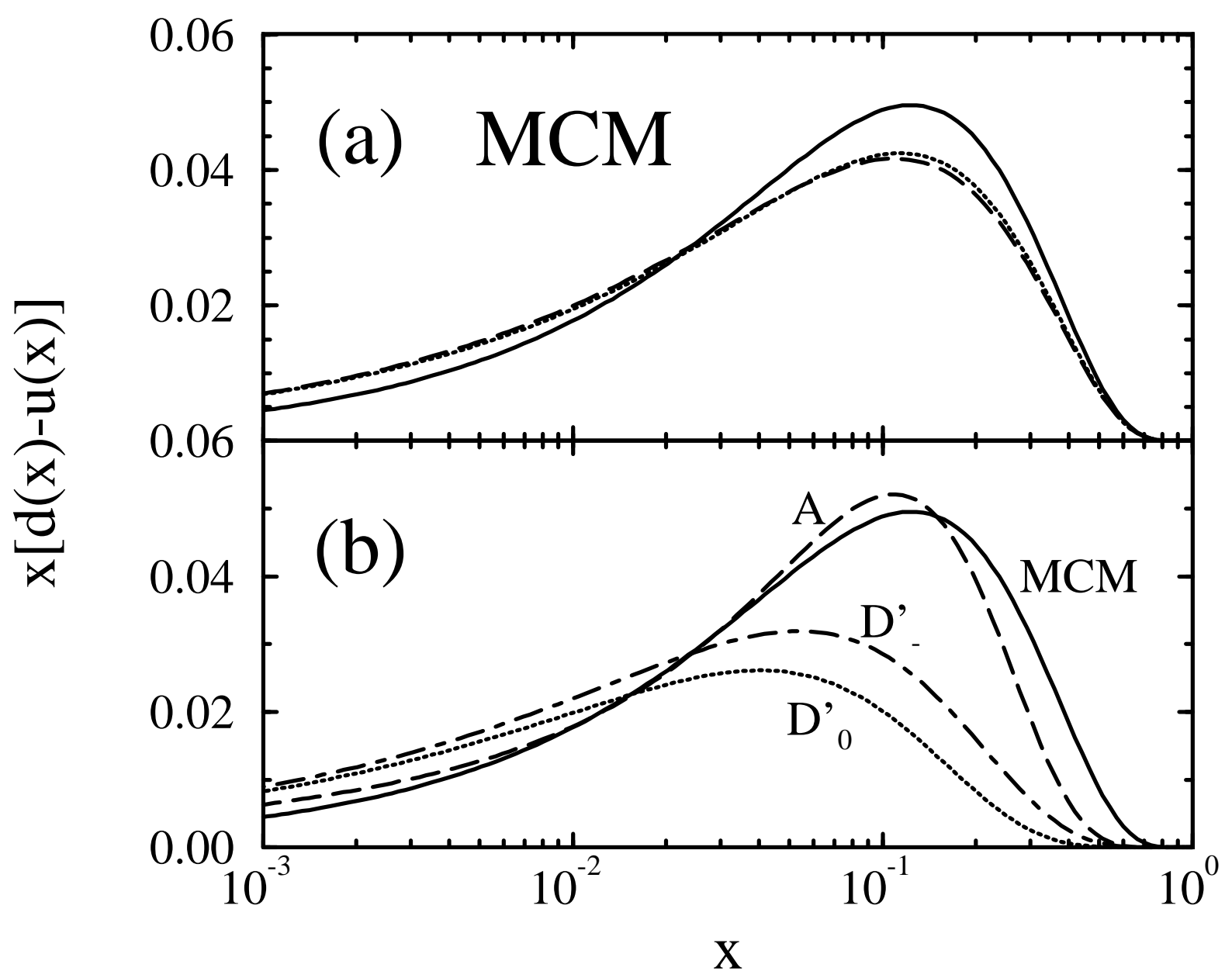




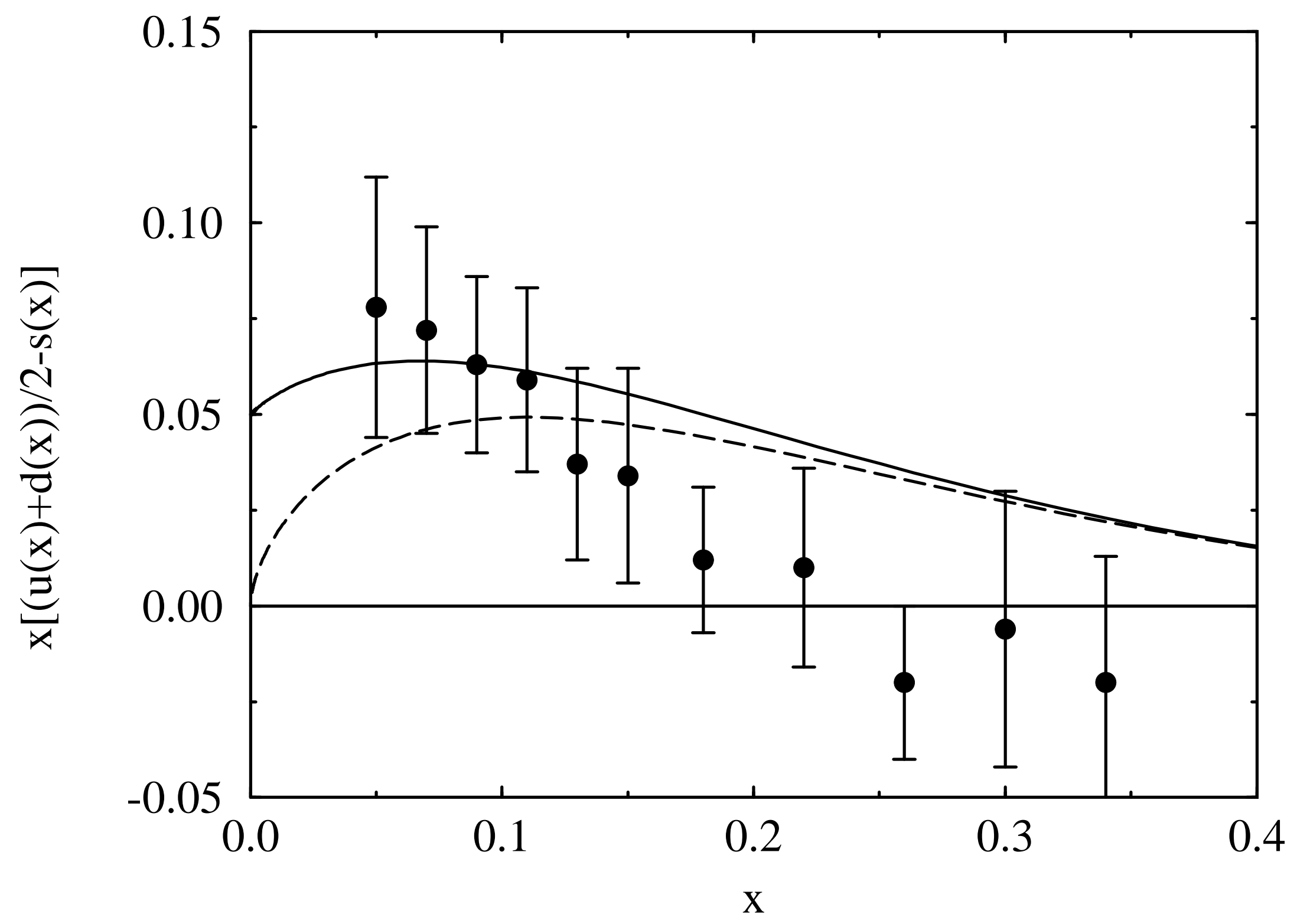




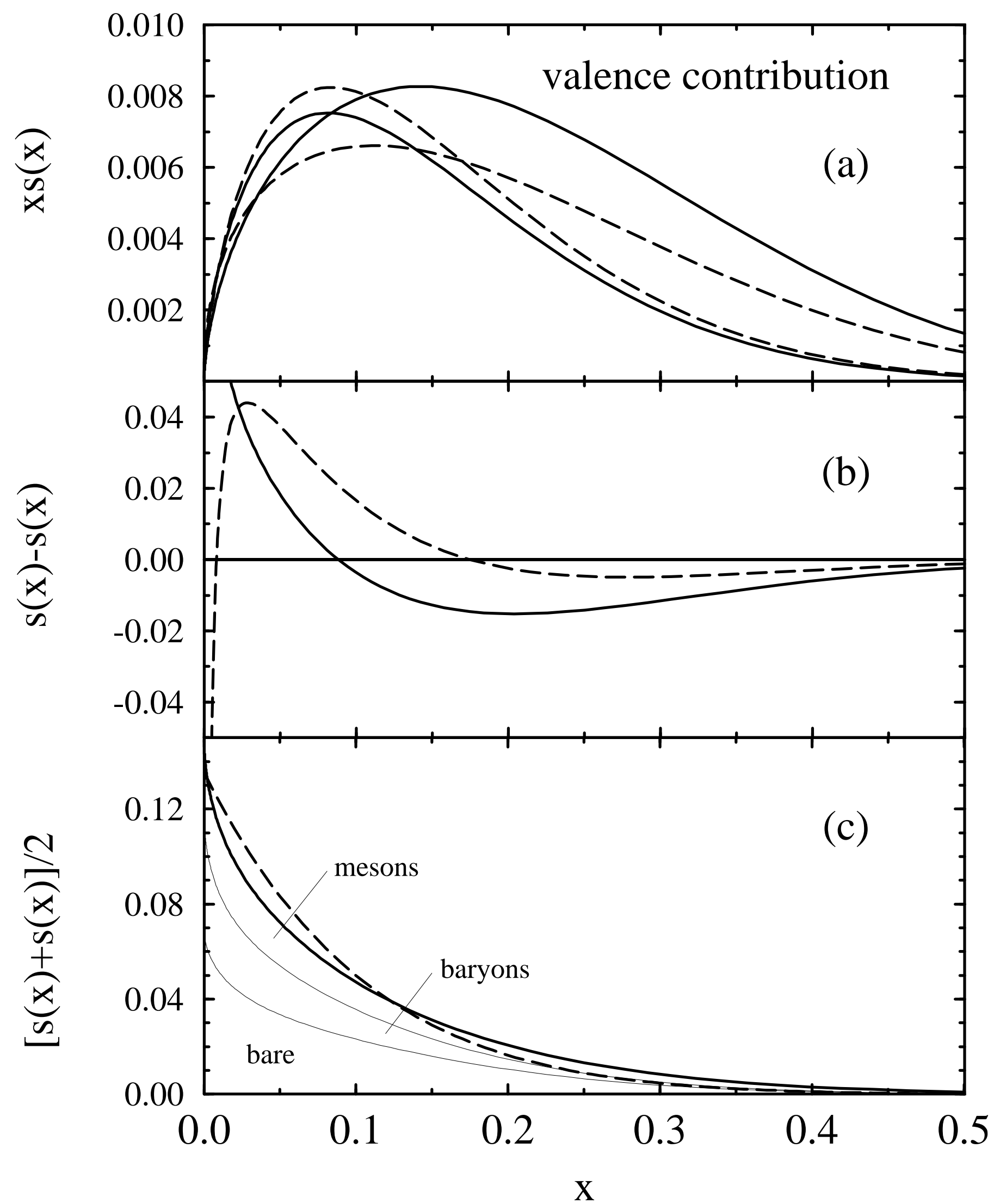

